\title{
Analytical model of mixed electroosmotic/pressure driven three immiscible fluids in a rectangular microchannel
}

\author{
Li Haiwang, Teck Neng Wong*, Nam-Trung Nguyen \\ School of Mechanical and Aerospace, Nanyang Technological University, 50 Nanyang, Avenue, \\ Singapore 639798, Singapore \\ * Corresponding author. Tel.: +65 67905587; fax: +65 67911859. E-mail address: \\ mtnwong@ntu.edu.sg (T.N. Wong).
}

\begin{abstract}
This paper presents a mathematical model to describe a three-fluid electroosmotic focusing/pumping techniques, in which an electrically non conducting fluid is focused and delivered by the combined interfacial viscous force of two conducting fluids and pressure gradient. The two conducting fluids are driven by electroosmosis and pressure gradient. The electrical potential in the two conducting fluids and the velocity distribution of the steady threefluid electroosmotic stratified flow in a rectangular microchannel were presented by assuming a planar interface between the three immiscible fluids. The effects of viscosity ratio, electroosmosis and pressure gradient on velocity profile and flow rate are analyzed to show the potential feasibility of this technique.
\end{abstract}

Keywords: Three-fluid stratified flow; Electrical double layer; Electroosmotic focusing

\section{Introduction}

In microfluidic devices, the flow focusing technique provides a particularly effective means of controlling the passage of chemical reagents or bio-samples in a microchannel network.

Hydrodynamic and electrokinetic focusing techniques are two popular flow focusing techniques. Stiles et al. [1] proposed a simple method to focus the sample stream by using either 
a single suction pump or capillary pumping effect. The focused stream width was controlled by varying the relative resistances of the side and inlet channel flows. Precise control of the focused sample stream width is crucial for different applications. For example, in cell counting and sorting applications performed in micromachined-based flow cytometers, the width of the focused stream should be at the same order of magnitude as that of the cell size [2, 3]. In addition, several studies [4] showed that the focused sample stream can be precisely guided and positioned by adjusting the relative flowrates of the two neighboring sheath flows. Lee et al. [4] applied flow focusing to develop various valveless microflow switches. However, the disadvantage of the proposed designs for pressure driven flow required a high flowrate ratio between the sheath and sample fluids to move the interface location or to switch the sample fluid. More recently, electroosmotic force was introduced to achieve switching $[5,6]$.

Since the surface-to-volume ratio in microscale is large and electroosmotic flow (EOF) is governed by surface charge, EOF would be more efficient than ordinary pressure driven flows [7]. This feature was exploited by EOF pumps. EOF pumps are popular since they contain no moving parts and are relatively easy to integrate in microfluidic circuits during fabrication [8]. Lin et al. [9] reported a numerical model for electrokinetic control, which can adjust the volume of the sample fluid. Fu et al. [10, 11] presented experimental and numerical results of electrokinetic flow injection. By applying different voltages at different parts of the channel, the sample fluid could be directed into a specific outlet channel. Gao et al. [12,13] derived the analytical solution of velocity profile and flowrate of two-liquid flow in microchannel which was driven both by electroosmotic force and pressure gradient.

While most of the previous theoretical studies mainly consider pressure alone for the threefluid flow $[4,14]$ or the combined effects of pressure gradient and electro-osmosis for two-liquid flow [12], there is few models to discuss about the focusing effect which takes into account of the combined effects of pressure gradient and electroosmosis. The present work proposes a theoretical model of three-fluid flow under conditions of electroosmosis, pressure gradient and the surface charge at the interfaces.

Fig. 1(a) shows the model of the three-fluid flow, two fluids (fluids 1 and 3) are conducting fluids with high electroosmotic mobility, while the focused fluid (fluid 2) is non conducting with a low electroosmotic mobility. For a given pressure gradient, different electric fields are applied 
across the conducting fluids, electroosmotic forces will be generated and the velocity of conducting fluids can be regulated depending on the directions and the strength of the applied electric fields. The fluid with low electroosmotic mobility is focused and delivered by the interfacial viscous force of the conducting fluids.

This paper aims to provide a theoretical analysis of the three fluids driven by the combined electroosmosis and pressure gradient. Analytical solutions of the EDL in the conducting fluid and velocities of three fluids are obtained in the fully developed section in a rectangular channel. The flow of the three fluids depends on the coupling effects of the three fluids which involve the pressure gradient and electrokinetic driven forces and the interfacial phenomenon. The model accounts for surface charges at the two liquid-liquid interfaces.

\begin{tabular}{|c|c|c|c|}
\hline \multicolumn{4}{|c|}{ Nomenclature } \\
\hline \multicolumn{2}{|c|}{$\alpha_{1}, \alpha_{2}, \alpha_{3}$ liquid fractions } & $\varepsilon$ & the permittivity of the dielectric \\
\hline$e$ & elementary charge, $e=1.602 \times 10^{-9}[\mathrm{C}]$ & $\varepsilon_{0}$ & the permittivity of the dielectric of vacuum \\
\hline E & the electric field & $\varepsilon_{r}$ & the dielectric constant comparing with $\varepsilon_{0}$ \\
\hline G & $\begin{array}{l}\text { parameter measuring the electroosmotic force by exter- } \\
\text { nal electric field }\end{array}$ & $\begin{array}{l}\psi \\
\mu\end{array}$ & $\begin{array}{l}\text { the electric potential } \\
\text { dynamic viscosity }\end{array}$ \\
\hline$h$ & height of the microchannel $[\mathrm{m}]$ & $\kappa$ & Debye-Hückel parameter $\left[\mathrm{m}^{-1}\right]$ \\
\hline$L_{\text {ref }}$ & the length scale & $\rho_{q}^{s}$ & the surface charge density \\
\hline M & electrokinetic effect in the matching conditions & $\beta^{4}$ & dynamic viscosity ratios \\
\hline \multirow{2}{*}{$\begin{array}{l}n_{0} \\
n_{i}\end{array}$} & ionic number concentration in the bulk $\left[\mathrm{m}^{-3}\right]$ & & \\
\hline & $\begin{array}{l}\text { ionic number concentration of the type-i in the bulk } \\
{\left[\mathrm{m}^{-3}\right]}\end{array}$ & $\begin{array}{l}\text { Sul } \\
\text { ref }\end{array}$ & reference quantity \\
\hline$k_{b}$ & Boltzmann constant, $k=1.381 \times 10^{-23}\left[\mathrm{JK}^{-1}\right]$ & 1 & conducting fluid 1 \\
\hline$K$ & electrokinetic parameter & 2 & non-conducting fluid 2 \\
\hline$p$ & pressure & 3 & conducting fluid 3 \\
\hline$q$ & flowrate & $q$ & charge of bulk \\
\hline \multicolumn{4}{|c|}{$\mathbf{r} \quad$ position vector $[\mathrm{m}]$} \\
\hline $\operatorname{Re}$ & Reynolds number & \multicolumn{2}{|c|}{ Superscripts } \\
\hline$t$ & time $[s]$ & 0 & fundamental state \\
\hline$T$ & temperature $[K]$ & - & dimensionless parameter \\
\hline$u$ & velocity & $p$ & pressure driven \\
\hline$U_{\text {ref }}$ & the velocity scale & $E$ & electroosmotic effect \\
\hline $\mathrm{v}$ & the velocity vector & , & derivative \\
\hline$w$ & half of width of the channel [m] & I & integrate \\
\hline \multicolumn{2}{|c|}{ Greek symbols } & \multicolumn{2}{|c|}{ Operators } \\
\hline & the density of charge & $\nabla$ & gradient \\
\hline & $\begin{array}{l}\text { electrostatic potential } \\
\text { zeta potential }\end{array}$ & $\partial$ & partial differential \\
\hline
\end{tabular}

\section{Theoretical model}

\subsection{Electric double layers in the conducting fluid and surface electric charges at the interface}

To analyze the system proposed above, a Cartesian coordinate system (x, y, z) is used where the origin point, $\mathrm{O}$, is set to be at the centre of the non conducting fluid and the symmetric line is shown in Fig. 1(b). Planar interfaces are assumed. The heights of the conducting fluids and of the non conducting fluid are denoted as $h_{1}, h_{3}$ and $2 h$, respectively. Half of the width of the channel is denoted by w. The aspect ratio is defined as $\chi=\left(h_{1}+h_{3}+2 h\right) / 2 w$. As a result, of surface 
charges, electric double layers (EDLs) form next to the two liquid-liquid interfaces and the channel walls that are in contact with the conducting fluid. For a more general situation, the walls of the microchannel may be made of different materials, so that the zeta potentials at the bottom and top walls are denoted as $\xi_{1}, \xi_{4}$, and at the side walls as $\xi_{2}, \xi_{5}$, respectively. The zeta potentials at the interfaces are $\xi_{3}$ and $\xi_{6}$. The electroosmotic flows are along the $\mathrm{x}$-direction. Due to symmetry, only half of the cross section $(z>0)$ of the rectangular channel is considered.

The three fluids are driven by the combined pressure and electroosmotic body forces. When the three-fluid flow is fully developed, the velocities of the three liquids, $\mathrm{u}_{1}, \mathrm{u}_{2}$ and $\mathrm{u}_{3}$ at position $\mathrm{r}$ along the channel are independent of x. The subscripts, 1, 2 and 3, denote the conducting fluid 1, non conducting fluid 2 and the conducting fluid 3 , respectively.

In this case, the conducting fluids are considered as symmetric electrolyte. The electric potentials in the conducting fluids due to the charged walls were taken as $\psi_{1}$ and $\psi_{3}$, respectively. The net charge densities in the two conducting fluids are $\rho_{\mathrm{q} 1}$ and $\rho_{\mathrm{q} 3}$. The length scale and velocity scale of the flow are taken as $\mathrm{L}_{\text {ref }}$ and $\mathrm{V}_{\text {ref }}$, respectively. The independent variable $\mathrm{r}$ and dependent variables $\mathrm{u}, \mathrm{p}, \psi$ and $\rho_{\mathrm{q}}$ are expressed in terms of the corresponding dimensionless quantities (shown with an overbar) by

$$
\left\{\begin{array}{l}
\mathbf{r}=L_{\text {ref }} \overline{\mathbf{r}} \\
u=U_{\text {ref }} \bar{u} \\
\psi=k_{b} T \bar{\psi} /\left(z_{0} e\right) \\
p=\rho_{1} U_{\text {ref }}^{2} \bar{p} \\
\rho_{q}=2 n_{0} z_{0} e \bar{\rho}_{q}
\end{array}\right.
$$

where $\rho$ is the liquid density, $\mathrm{k}_{\mathrm{b}}$ is Boltzmann constant, $\mathrm{T}$ is the absolute temperature, $\mathrm{z}_{0}$ is the valence of the ions, e is elementary charge, and $n_{0}$ is the reference value of the ion concentration.

The fluid fractions of fluids 1, 2 and 3 are defined as $\alpha_{1}=\frac{h_{1}}{h_{1}+2 h+h_{3}}, \alpha_{2}=\frac{2 h}{h_{1}+2 h+h_{3}}$ and $\alpha_{3}=\frac{h_{3}}{h_{1}+2 h+h_{3}}$, respectively.

The electric potential in the conducting fluids is first considered. Assuming that the electric charge density is not affected by the external electric field due to thin EDLs and the small fluid velocity, the charge convection can be ignored and the electric field equation and the fluid flow equation are decoupled $[15,16]$. Based on the assumption of local thermodynamic equilibrium, 
for a small zeta potential, the electric potentials due to the charged wall are described by the linear Poisson-Boltzmann equation which can be written in terms of dimensionless variables as

$$
\nabla^{2} \bar{\psi}=K^{2} \bar{\psi}
$$

where $\mathrm{K}=\mathrm{L}_{\text {ref }} \mathrm{K}$ is the ratio of the length scale $\mathrm{L}_{\text {ref }}$ to the characteristic double-layer thickness $1 / \kappa$. For this case, the reference length is chosen as $\mathrm{L}_{\mathrm{ref}}=\mathrm{w}$. Here, $\kappa$ is the Debye-Hückel parameter

$$
\frac{1}{\kappa}=\left(\frac{\varepsilon k_{b} T}{2 z_{0}^{2} e^{2} n_{0}}\right)^{\frac{1}{2}}
$$

where $\varepsilon$ is the permittivity of the conducting fluid. Based on the linear approximation, the dimensionless volumetric charge density is given by

$$
\bar{\rho}_{q}=-\bar{\psi}(\bar{z}, \bar{y}) .
$$

Due to the symmetry of the EDL fields in the rectangular channel, Eq. (2) is subjected to the following boundary conditions:

$$
\begin{aligned}
& \text { (conducting fluid 1) }\left\{\begin{array}{lll}
\partial \bar{\psi}_{1} / \partial \bar{z}=0 & \text { at } & \bar{z}=0 \\
\bar{\psi}_{1}=\bar{\xi}_{2} & \text { at } & \bar{z}=\bar{w} \\
\bar{\psi}_{1}=\bar{\xi}_{3} & \text { at } & \bar{y}=-\bar{h} \\
\bar{\psi}_{1}=\bar{\xi}_{1} & \text { at } & \bar{y}=-\bar{h}_{1}-\bar{h}
\end{array}\right. \\
& \text { (conducting fluid 3) }\left\{\begin{array}{lll}
\partial \bar{\psi}_{3} / \partial \bar{z}=0 & \text { at } & \bar{z}=0 \\
\bar{\psi}_{3}=\bar{\xi}_{5} & \text { at } & \bar{z}=\bar{w} \\
\bar{\psi}_{3}=\bar{\xi}_{6} & \text { at } & \bar{y}=\bar{h} \\
\bar{\psi}_{3}=\bar{\xi}_{4} & \text { at } & \bar{y}=\bar{h}_{3}+\bar{h}
\end{array}\right.
\end{aligned}
$$

The solutions to the Poisson-Boltzmann equation subjected to the above boundary conditions are obtained as

$$
\begin{aligned}
\bar{\psi}_{1}= & \sum_{j=0}^{\infty} \frac{4 \bar{\xi}_{1}}{(2 j+1) \pi}(-1)^{j+1} \frac{\sinh [(\bar{h}+\bar{y})]}{\sinh \left(B_{j} \bar{h}_{1}\right)} \cos \left(\lambda_{j} \bar{z}\right) \\
& -\sum_{j=0}^{\infty} \frac{4 \bar{\xi}_{3}}{(2 j+1) \pi}(-1)^{j+1} \times \frac{\sinh \left[B_{j}\left(\bar{h}_{1}+\bar{h}+\bar{y}\right)\right]}{\sinh \left[B_{j} \bar{h}_{1}\right]} \times \cos \left(\lambda_{j} \bar{z}\right) \\
& +\sum_{p=1}^{\infty} \frac{2 \bar{\xi}_{2}}{p \pi}\left[(-1)^{p}-1\right] \frac{\cosh \left(A_{p 1} \bar{z}\right)}{\cosh \left(A_{p 1} \bar{w}\right)} \sin \left[\frac{p \pi}{\bar{h}_{1}}(\bar{y}+\bar{h})\right]
\end{aligned}
$$

for conducting fluid 1 and 


$$
\begin{aligned}
\bar{\psi}_{3}= & -\sum_{j=0}^{\infty} \frac{4 \bar{\xi}_{4}}{(2 j+1) \pi} \times(-1)^{j+1} \times \frac{\sinh \left[B_{j}(\bar{y}-\bar{h})\right]}{\sinh \left(B_{j} \bar{h}_{3}\right)} \cos \left(\lambda_{j} \bar{z}\right) \\
& +\sum_{j=0}^{\infty} \frac{4 \bar{\xi}_{6}}{(2 j+1) \pi} \times(-1)^{j+1} \times \frac{\sinh \left[B_{j}\left(\bar{y}-\bar{h}_{3}-\bar{h}\right)\right]}{\sinh \left[B_{j} \bar{h}_{3}\right]} \cos \left(\lambda_{j} \bar{z}\right) \\
& -\sum_{p=1}^{\infty} \frac{2 \bar{\xi}_{5}}{p \pi}\left[(-1)^{p}-1\right] \frac{\cosh \left(A_{p 3} \bar{z}\right)}{\cosh \left(A_{p 3} \bar{w}\right)} \sin \left[\frac{p \pi}{\bar{h}_{3}}(\bar{y}-\bar{h})\right]
\end{aligned}
$$

for conducting fluid 3, where

$$
\left\{\begin{array}{c}
\lambda_{j}=\frac{(2 j-1) \pi}{2 \bar{w}} \\
B_{j}=\sqrt{K^{2}+\lambda_{j}^{2}} \\
A_{p 1}=\sqrt{K^{2}+\left(\frac{p \pi}{h_{1}}\right)^{2}} \\
A_{p 3}=\sqrt{K^{2}+\left(\frac{p \pi}{h_{3}}\right)^{2}}
\end{array}\right.
$$

In the above discussion of electroosmosis, the charge state of the surface is described in terms of surface potential at the shear plane, which is identified by the zeta potential [17]. This surface potential is related to the charge density at the surface [18]. From electrostatics, the normal component of the gradient of the electric potential, $\psi$, jumps by an amount proportional to the surface charge density, $\rho_{q}^{s}$. That is

$$
\rho_{q}^{s}=-\varepsilon \frac{\partial \psi}{\partial y}
$$

It is assumed that the gradient of electric potential in the non conducting fluid vanishes. Using the reference surface charge density as $\left(\varepsilon k_{b} T\right) /\left(L_{r e f} z_{0} e\right)$, we obtain the dimensionless surface charge densities at the liquid-liquid interface as

$$
\begin{aligned}
\bar{\rho}_{q 1}^{s}(\bar{z})= & -\frac{\partial \bar{\psi}_{1}(-\bar{h}, \bar{z})}{\partial \bar{y}} \\
= & -\sum_{j=1}^{\infty} \frac{4(-1)^{j+1} B_{j}}{(2 j+1) \pi}\left[\frac{\bar{\xi}_{1}}{\sinh \left(B_{j} \bar{h}_{1}\right)}-\frac{\bar{\xi}_{3}}{\tanh \left(B_{j} \bar{h}_{1}\right)}\right] \cos \left(\lambda_{j} \bar{z}\right) \\
& -\sum_{p=1}^{\infty} \frac{2 \bar{\xi}_{2}}{\bar{h}_{1}}\left[(-1)^{p}-1\right] \frac{\cosh \left(A_{p 1} \bar{z}\right)}{\cosh \left(A_{p 1} \bar{w}\right)}
\end{aligned}
$$

for the surface charge at interface $1-2$, and 


$$
\begin{aligned}
\bar{\rho}_{q 3}^{s}(\bar{z})= & -\frac{\partial \bar{\psi}_{3}(\bar{h}, \bar{z})}{\partial \bar{y}} \\
= & \sum_{j=1}^{\infty} \frac{4(-1)^{j+1} B_{j}}{(2 j+1) \pi}\left[\frac{\bar{\xi}_{4}}{\sinh \left(B_{j} \bar{h}_{3}\right)}-\frac{\bar{\xi}_{6}}{\tanh \left(B_{j} \bar{h}_{3}\right)}\right] \cos \left(\lambda_{j} \bar{z}\right) \\
& +\sum_{p=1}^{\infty} \frac{2 \bar{\xi}_{5}}{\bar{h}_{3}}\left[(-1)^{p}-1\right] \frac{\cosh \left(A_{p 3} \bar{z}\right)}{\cosh \left(A_{p 3} \bar{w}\right)}
\end{aligned}
$$

The solutions of Eqs. (11) and (12) show that the contributions of zeta potential at the top/bottom walls, $\bar{\xi}_{4}$ and $\bar{\xi}_{1}$, are relatively small and the contributions of side walls, $\bar{\xi}_{2}$ and $\bar{\xi}_{5}$ are also relatively small except when $\mathrm{z}$ approaches to $\mathrm{w}$. The volumetric net charge density, Eq. (4), and the interface charge density, Eqs. (11) and (12), are required to determine the electrostatic force caused by the presence of zeta potential. The bulk electrostatic force is considered as an additional body force exerting on the conducting fluid in the conventional Navier-Stokes equation. Therefore, the conducting fluids are under the action of pressure gradient, electrostatic force and the viscous shear force at the interface. Similarly, the non conducting fluid flows as a result of the pressure gradient and the external electrostatic force due to the electrokinetic charge density at the interface, which will be discussed in the following section.

\subsection{Momentum equations of the three-fluid flow}

The dimensionless momentum equation for an incompressible Newtonian liquid is given by

$$
\frac{\partial(\bar{\rho} \bar{v})}{\partial \bar{t}}+\nabla \cdot(\bar{\rho} \overline{\mathbf{v}} \overline{\mathbf{v}})=-\nabla \bar{p}+\mathbf{G} \bar{\rho}_{q}+\frac{1}{\operatorname{Re}} \bar{\mu} \nabla^{2} \overline{\mathbf{v}}
$$

To evaluate the electrokinetic effects, our model assumes that the flow is formed by three simple immiscible Newtonian liquids with constant viscosities, which are independent of shear rate and the local electric field strength. The model assumes:

(1) All the three liquids are Newtonian and incompressible.

(2) The properties of the liquids are independent of local electric field and ion concentration. The electric field strength and ion concentration may affect the properties of the conducting fluids. In the current study, these effects are neglected [19]. 
(3) The liquid's properties are independent of temperature. Joule heating is neglected for dilute electrolytes and low field strength [20].

(4) The flow is fully developed with the non-slip boundary condition. The second term on the left-hand side of the Eq. (13), $\nabla \cdot(\bar{\rho} \bar{v} \bar{v})$, will be vanished.

(5) The pressure gradient is assumed to be uniform along the channel, and the pressure gradients along $\mathrm{y}$ - and z-directions are both zero.

Because EDLs only form in the conducting fluids, the momentum equations of the three liquids reduce to

$$
\frac{\partial\left(\bar{u}_{1}\right)}{\partial \bar{t}}=\frac{1}{\operatorname{Re}}\left(\frac{\partial^{2} \bar{u}_{1}}{\partial \bar{y}^{2}}+\frac{\partial^{2} \bar{u}_{1}}{\partial \bar{z}^{2}}\right)+G_{x 1} \bar{\rho}_{q 1}-\frac{d \bar{p}}{d \bar{x}}
$$

for conducting fluid 1 ,

$$
\bar{\rho}_{3} \frac{\partial\left(\bar{u}_{3}\right)}{\partial \bar{t}}=\frac{\bar{\mu}_{3}}{\operatorname{Re}}\left(\frac{\partial^{2} \bar{u}_{3}}{\partial \bar{y}^{2}}+\frac{\partial^{2} \bar{u}_{3}}{\partial \bar{z}^{2}}\right)+G_{x 3} \bar{\rho}_{q 3}-\frac{d \bar{p}}{d \bar{x}}
$$

for the conducting fluid 3 , and

$$
\bar{\rho}_{2} \frac{\partial\left(\bar{u}_{2}\right)}{\partial \bar{t}}=\frac{\bar{\mu}_{2}}{\operatorname{Re}}\left(\frac{\partial^{2} \bar{u}_{2}}{\partial \bar{y}^{2}}+\frac{\partial^{2} \bar{u}_{2}}{\partial \bar{z}^{2}}\right)-\frac{d \bar{p}}{d \bar{x}}
$$

for the non conducting fluid 2, where $G_{x 1}=\frac{2 z_{0} e n_{0} L_{r e f} E_{x 1}}{\rho_{\text {ref }} U_{\text {ref }}^{2}}$ and $G_{x 3}=\frac{2 z_{0} e n_{0} L_{\text {ref }} E_{x 3}}{\rho_{\text {ref }} U_{\text {ref }}^{2}}$. Here, the reference viscosity and the density are those of the conducting fluid 1 as $\mu_{\text {ref }}=\mu_{1}$ and $\rho_{\text {ref }}=\rho_{1}$. Thus, $\bar{\mu}_{1}=\beta_{1}=1, \bar{\mu}_{2}=\beta_{2}=\frac{\mu_{2}}{\mu_{1}}$ and $\bar{\mu}_{3}=\beta_{3}=\frac{\mu_{3}}{\mu_{1}}$ are the dynamic viscosity ratios. The continuity conditions of the velocities at the liquid/liquid interfaces are:

$$
\begin{array}{ll}
u_{1}=u_{2}, & \text { at } \quad y=-h \quad \text { (interface } 1-2) \\
u_{2}=u_{3}, & \text { at } \quad y=h \quad \text { (interface } 2-3)
\end{array}
$$

The shear stress balances, which jumps abruptly at the interface due to the presence of a certain surface charge density, 


$$
\begin{aligned}
& \mu_{1} \frac{\partial u_{1}}{\partial y}+E_{x 1} \rho_{q}^{s}=\mu_{2} \frac{\partial u_{2}}{\partial y}, \quad \text { at } y=-h \quad(\text { interface } 1-2) \\
& \mu_{3} \frac{\partial u_{3}}{\partial y}+E_{x 3} \rho_{q}^{s}=\mu_{2} \frac{\partial u_{2}}{\partial y}, \quad \text { at } y=h \quad(\text { interface } 2-3)
\end{aligned}
$$

Here $\mathrm{y}$ is the direction normal to the interface of the two liquids. As planar interface is assumed, the normal direction of interface is along the y-direction. The dimensionless matching conditions become

$$
\begin{aligned}
& \text { (interface } 1-2)\left\{\begin{array}{l}
\bar{u}_{1}(\bar{z},-\bar{h}, \bar{t})=\bar{u}_{2}(\bar{z},-\bar{h}, \bar{t}) \\
\frac{\partial \bar{u}_{1}(\bar{z},-\bar{h}, \bar{t})}{\partial \bar{y}}=\beta_{2} \frac{\partial \bar{u}_{2}(\bar{z},-\bar{h}, \bar{t})}{\partial \bar{y}}-M_{1} \bar{\rho}_{q 1}^{s}(\bar{z})
\end{array}\right. \\
& \text { (interface } 2-3)\left\{\begin{array}{l}
\bar{u}_{3}(\bar{z}, \bar{h}, \bar{t})=\bar{u}_{2}(\bar{z}, \bar{h}, \bar{t}) \\
\frac{\partial \bar{u}_{3}(\bar{z}, \bar{h}, \bar{t})}{\partial \bar{y}}=\left(\beta_{2} / \beta_{3}\right) \frac{\partial \bar{u}_{2}(\bar{z}, \bar{h}, \bar{t})}{\partial \bar{y}}-M_{3} \bar{\rho}_{q 3}^{s}(\bar{z})
\end{array}\right.
\end{aligned}
$$

where $M_{1}=\frac{\varepsilon k_{b} T E_{x 1}}{z_{0} U_{\text {ref }} \mu_{\text {ref }}}$ and $M_{3}=\frac{\varepsilon k_{b} T E_{x 3}}{z_{0} e U_{\text {ref }} \mu_{\text {ref }}}$.

In the rectangular-cross-section channel, the dimensionless boundary conditions for fluids 1,2 and 3 are respectively,

$$
\begin{aligned}
& \text { (conducting fluid 1) } \begin{cases}\frac{\partial \bar{u}_{1}}{\partial \bar{z}}=0 & \text { when } \bar{z}=0 \\
\bar{u}_{1}=0 & \text { when } \bar{z}=\bar{w} \\
\bar{u}_{1}=0 & \text { when } \bar{y}=-\bar{h}-\bar{h}_{1} \\
\bar{u}_{1}=0 & \text { when } \bar{t}=0\end{cases} \\
& \text { (non conducting fluid 2) } \begin{cases}\frac{\partial \bar{u}_{2}}{\partial \bar{z}}=0 & \text { when } \bar{z}=0 \\
\bar{u}_{2}=0 & \text { when } \bar{t}=0\end{cases} \\
& \text { (conducting fluid 3) } \begin{cases}\frac{\partial \bar{u}_{3}}{\partial \bar{z}}=0 & \text { when } \bar{z}=0 \\
\bar{u}_{3}=0 & \text { when } \bar{z}=\bar{w} \\
\bar{u}_{3}=0 & \text { when } \bar{y}=\bar{h}_{3}+\bar{h} \\
\bar{u}_{2}=0 & \text { when } \bar{t}=0\end{cases}
\end{aligned}
$$

Due to linearity, the velocity of the conducting fluids and of the non-conducting fluid in Eqs. (14)-(16) can be decomposed into two parts:

$$
\bar{u}=\bar{u}^{p}+\bar{u}^{E}
$$

where $\bar{u}^{E}$ corresponds to the velocity driven by electroosmotic force, and $\bar{u}^{p}$ is the velocity driven by pressure gradient. The final velocities are the superposition of these electroosmotic and pressure-driven components 


$$
\left\{\begin{array}{l}
\bar{u}_{1}=\bar{u}_{1}^{p}+\bar{u}_{1}^{E} \\
\bar{u}_{2}=\bar{u}_{2}^{p}+\bar{u}_{2}^{E} \\
\bar{u}_{3}=\bar{u}_{3}^{p}+\bar{u}_{3}^{E}
\end{array}\right.
$$

\subsection{Velocity fields of the three liquids}

For the steady state fully developed flow, the dimensionless velocity for the conducting fluid 1, when Eq. (14) is combined with Eq. (4), becomes

$$
\frac{\partial^{2} \bar{u}_{1}^{E}}{\partial \bar{z}^{2}}+\frac{\partial^{2} \bar{u}_{1}^{E}}{\partial \bar{y}^{2}}=\operatorname{Re}_{x 1} \bar{\psi}_{1}(\bar{z}, \bar{y})
$$

corresponding to the velocity component driven by the electroosmotic force and

$$
\frac{\partial^{2} \bar{u}_{1}^{P}}{\partial \bar{z}^{2}}+\frac{\partial^{2} \bar{u}_{1}^{P}}{\partial \bar{y}^{2}}=\operatorname{Re} \frac{d \bar{p}}{d \bar{x}}
$$

corresponding to the velocity component driven by the pressure gradient. Similarly, the velocities of the non conducting fluid 2 and the conducting fluid 3 are written in two components as

$$
\frac{\partial^{2} \bar{u}_{2}^{E}}{\partial \bar{z}^{2}}+\frac{\partial^{2} \bar{u}_{2}^{E}}{\partial \bar{y}^{2}}=0
$$

and

$$
\frac{\partial^{2} \bar{u}_{3}^{E}}{\partial \bar{z}^{2}}+\frac{\partial^{2} \bar{u}_{3}^{E}}{\partial \bar{y}^{2}}=-\frac{\operatorname{Re}}{\beta_{3}} G_{x 3} \bar{\rho}_{q 3}
$$

corresponding to the velocity component influenced by the electroosmotic flow and

$$
\begin{aligned}
& \frac{\partial^{2} \bar{u}_{2}^{P}}{\partial \bar{z}^{2}}+\frac{\partial^{2} \bar{u}_{2}^{P}}{\partial \bar{y}^{2}}=\operatorname{Re} \frac{d \bar{p}}{d \bar{x}} \frac{1}{\beta_{2}} \\
& \frac{\partial^{2} \bar{u}_{3}^{P}}{\partial \bar{z}^{2}}+\frac{\partial^{2} \bar{u}_{3}^{P}}{\partial \bar{y}^{2}}=\frac{\operatorname{Re}}{\beta_{3}} \frac{d \bar{p}}{d \bar{x}}
\end{aligned}
$$

corresponding to the velocity component driven by the pressure gradient.

Using the separation of variables method and substituting the solution of $\bar{\psi}_{1}$ from Eq. (7), the analytical velocity profile corresponding to the electroosmotic force, $\bar{u}_{1}^{E}$, and velocity profile 
corresponding to the pressure gradient, $\bar{u}_{11}^{p}$, are obtained from Eqs. (27) and (28), respectively. They are

$$
\begin{aligned}
& \bar{u}_{1}^{E}(\bar{y}, \bar{z})=\sqrt{\frac{2}{\bar{w}}} \sum_{j=0}^{\infty}\left\{\left\{\tanh \left[\lambda_{j}\left(\bar{h}+\bar{h}_{1}\right)\right] \cosh \left(\lambda_{j} \bar{y}\right)+\sinh \left(\lambda_{j} \bar{y}\right)\right\} b_{1 j}^{E}\right. \\
& \left.-\frac{\phi_{1 j}\left(-\bar{h}-\bar{h}_{1}\right) \cosh \left(\lambda_{j} \bar{y}\right)}{\cosh \left[\lambda_{j}\left(\bar{h}+\bar{h}_{1}\right)\right]}+\phi_{1 j}(\bar{y})\right\} \cos \left(\lambda_{j} \bar{z}\right) \\
& \bar{u}_{1}^{p}(\bar{y}, \bar{z})=\sqrt{\frac{2}{\bar{w}}} \sum_{j=0}^{\infty}\left\{\frac{1}{\lambda_{j}^{3}} \operatorname{Re} \frac{d \bar{p}}{d \bar{x}} \sqrt{\frac{2}{\bar{w}}}(-1)^{j}\left\{\frac{\cosh \left(\lambda_{j} \bar{y}\right)}{\cosh \left[\lambda_{j}\left(\bar{h}_{1}+\bar{h}\right)\right]}-1\right\}\right. \\
& \left.+\left\{\tanh \left[\lambda_{j}\left(\bar{h}_{1}+\bar{h}\right)\right] \cosh \left(\lambda_{j} \bar{y}\right)+\sinh \left(\lambda_{j} \bar{y}\right)\right\} b_{1 j}^{p}\right\} \cos \left(\lambda_{j} \bar{z}\right)
\end{aligned}
$$

The dimensionless velocity profiles for the non conducting fluid $2^{\left(\bar{u}_{2}^{E}, \bar{u}_{2}^{p}\right)}$, and the conducting fluid $3\left(\bar{u}_{3}^{E}, \bar{u}_{3}^{p}\right)$ are also obtained respectively as

$$
\begin{aligned}
& \bar{u}_{2}^{E}=\sqrt{\frac{2}{\bar{w}}} \sum_{0}^{\infty}\left[a_{2 j}^{E}(\bar{y}) \cosh \left(\lambda_{j} \bar{y}\right)+b_{2 j}^{E}(\bar{y}) \sinh \left(\lambda_{j} \bar{y}\right)\right] \cos \left(\lambda_{j} \bar{z}\right) \\
& \bar{u}_{2}^{p}=\sqrt{\frac{2}{\bar{w}}} \sum_{j=1}^{\infty}\left\{a_{2 j}^{p} \cosh \left(\lambda_{j} \bar{y}\right)+b_{2 j}^{p} \sinh \left(\lambda_{j} \bar{y}\right)-\frac{(-1)^{j}}{\beta_{2} \lambda_{j}^{3}} \operatorname{Re} \times \frac{d \bar{p}}{d \bar{x}} \times \sqrt{\frac{2}{\bar{w}}}\right\} \times \cos \left(\lambda_{j} \bar{z}\right) \\
& \bar{u}_{3}^{E}(\bar{y}, \bar{z})=\sqrt{\frac{2}{\bar{w}}} \sum_{j=0}^{\infty}\left\{\left\{-\tanh \left[\lambda_{j}\left(\bar{h}+\bar{h}_{3}\right)\right] \cosh \left(\lambda_{j} \bar{y}\right)+\sinh \left(\lambda_{j} \bar{y}\right)\right\} \cdot b_{3 j}^{E}\right. \\
& \left.-\frac{\phi_{3 j}\left(\bar{h}+\bar{h}_{3}\right)}{\cosh \left[\lambda_{j}\left(\bar{h}+\bar{h}_{3}\right)\right]} \cosh \left(\lambda_{j} \bar{y}\right)+\phi_{3 j}(\bar{y})\right\} \cos \left(\lambda_{j} \bar{z}\right) \\
& \bar{u}_{3}^{p}(\bar{y}, \bar{z})=\sqrt{\frac{2}{\bar{w}}} \sum_{j=0}^{\infty}\left\{\frac{1}{\lambda_{j}^{3}} \frac{\operatorname{Re}}{\beta_{3}} \frac{d \bar{p}}{d \bar{x}} \sqrt{\frac{2}{\bar{w}}}(-1)^{j}\left\{\frac{\cosh \left(\lambda_{j} \bar{y}\right)}{\cosh \left[\lambda_{j}\left(\bar{h}_{3}+\bar{h}\right)\right]}-1\right\}\right. \\
& \left.+\left\{-\tanh \left[\lambda_{j}\left(\bar{h}_{3}+\bar{h}\right)\right] \cosh \left(\lambda_{j} \bar{y}\right)+\sinh \left(\lambda_{j} \bar{y}\right)\right\} b_{3 j}^{P}\right\} \cos \left(\lambda_{j} \bar{z}\right)
\end{aligned}
$$

The detailed mathematical derivation of the coefficients $\phi_{1 j}(\bar{y})$ and $\phi_{3 j}(\bar{y})$ is presented in Appendix A.

The matching conditions given in Eqs. (21) and (22) can also be decomposed as 


$$
\begin{aligned}
& \text { (interface } 1 \text { - 2) }\left\{\begin{array}{l}
\bar{u}_{1}^{E}(\bar{z},-\bar{h}, \bar{t})=\bar{u}_{2}^{E}(\bar{z},-\bar{h}, \bar{t}) \\
\frac{\partial \bar{u}_{1}^{E}(\bar{z},-\bar{h}, \bar{t})}{\partial \bar{y}}=\beta_{2} \frac{\partial \bar{u}_{2}^{E}(\bar{z},-\bar{h}, \bar{t})}{\partial \bar{y}}-M_{1} \bar{\rho}_{q 1}^{s}(\bar{z})
\end{array}\right. \\
& \text { (interface } 1-2)\left\{\begin{array}{l}
\bar{u}_{1}^{p}(\bar{z},-\bar{h})=\bar{u}_{2}^{p}(\bar{z},-\bar{h}) \\
\frac{\partial \bar{u}_{1}^{p}(\bar{z},-\bar{h})}{\partial \bar{y}}=\beta_{2} \frac{\partial \bar{u}_{2}^{p}(\bar{z},-\bar{h})}{\partial \bar{y}}
\end{array}\right.
\end{aligned}
$$

and

$$
\begin{aligned}
& \text { (interface } 2 \text { - 3) }\left\{\begin{array}{l}
\bar{u}_{3}^{E}(\bar{z}, \bar{h})=\bar{u}_{2}^{E}(\bar{z}, \bar{h}) \\
\frac{\partial \bar{u}_{3}^{E}(\bar{z}, \bar{h})}{\partial \bar{y}}=\left(\beta_{2} / \beta_{3}\right) \frac{\partial \bar{u}_{2}^{E}(\bar{z}, \bar{h})}{\partial \bar{y}}-M_{3} \bar{\rho}_{q 3}^{s}(\bar{z})
\end{array}\right. \\
& \text { (interface } 2-3)\left\{\begin{array}{l}
\bar{u}_{3}^{p}(\bar{z}, \bar{h})=\bar{u}_{2}^{p}(\bar{z}, \bar{h}) \\
\frac{\partial \bar{u}_{3}^{p}(\bar{z}, \bar{h})}{\partial \bar{y}}=\left(\beta_{2} / \beta_{3}\right) \frac{\partial \bar{u}_{2}^{p}(\bar{z}, \bar{h})}{\partial \bar{y}}
\end{array}\right.
\end{aligned}
$$

At interface 1-2, substituting Eqs. (33) and (35) into Eq. (39) and substituting Eqs. (34) and (36) into Eq. (40); at inter-face 2-3, substituting Eqs. (35) and (37) into Eq. (41) and substituting Eqs. (36) and (38) into Eq. (42), we can obtain the constants

$$
\begin{aligned}
& a_{2 j}^{E}=J b_{1 j}^{E}+K b_{3 j}^{E}+L \\
& b_{3 j}^{E}=b_{2 j}^{E} / B+I \\
& b_{1 j}^{E}=b_{2 j}^{E} / A+H
\end{aligned}
$$

$$
\begin{aligned}
& b_{2 j}^{E}=(C I+D H+G) /[1-C / B-D / A] \\
& b_{2 j}^{p}=[O-R T / S-P U / V] /[1-R / S-P / V] \\
& b_{1 j}^{p}=b_{2 j}^{p} / S-T / S \\
& b_{3 j}^{P}=b_{2 j}^{P} / V-U / V \\
& a_{2 j}^{p}=N b_{3 j}^{P}+Q b_{1 j}^{p}+M
\end{aligned}
$$

The detailed mathematical derivation of the coefficients $\mathrm{A}$ to $\mathrm{V}, \phi_{1 j}^{\prime}, \phi_{3 j}^{\prime}$ is presented in Appendix B.

The contribution of surface charges, $\bar{\rho}_{q j 1}^{s}$ and $\bar{\rho}_{q j 3}^{s}$, can be obtained by applying Fourier transform to $\bar{\rho}_{q 1}^{s}$ and ${ }^{-} \bar{\rho}_{q 3}^{s}$, these are 


$$
\begin{aligned}
\bar{\rho}_{q j 1}^{s}= & -\sqrt{\frac{\bar{w}}{2}} \frac{4(-1)^{j+1} B_{j}}{(2 j+1) \pi}\left[\frac{\bar{\xi}_{1}}{\sinh \left(B_{j} \bar{h}_{1}\right)}-\frac{\bar{\xi}_{3}}{\tanh \left(B_{j} \bar{h}_{1}\right)}\right] \\
& -\sqrt{\frac{2}{\bar{w}}} \times \sum_{p=1}^{\infty} \frac{2 \bar{\xi}_{2}}{\bar{h}_{1}}\left[(-1)^{p}-1\right] \times \frac{(-1)^{j} \lambda_{j}}{A_{p 1}^{2}+\lambda_{j}^{2}} \\
\bar{\rho}_{q j 3}^{s}= & \sqrt{\frac{\bar{w}}{2}} \frac{4(-1)^{j+1} B_{j}}{(2 j+1) \pi}\left[\frac{\bar{\xi}_{4}}{\sinh \left(B_{j} \bar{h}_{3}\right)}-\frac{\bar{\xi}_{6}}{\tanh \left(B_{j} \bar{h}_{3}\right)}\right] \\
& +\sum_{p=1}^{\infty} \frac{2 \bar{\xi}_{5}}{\bar{h}_{3}}\left[(-1)^{p}-1\right] \times(-1)^{j} \times \frac{\lambda_{j}}{A_{p 3}^{2}+\lambda_{j}^{2}}
\end{aligned}
$$

The dimensionless volumetric flowrates through the rectangular-cross-section channel can be defined as $\bar{q}_{1}=\bar{q}_{1}^{E}+\bar{q}_{1}^{p}=q_{1} /\left(L_{\text {ref }}^{2} U_{\text {ref }}\right), \bar{q}_{2}=\bar{q}_{2}^{E}+\bar{q}_{2}^{p}=q_{3} /\left(L_{\text {ref }}^{2} U_{\text {ref }}\right) \quad$ and $\bar{q}_{3}=\bar{q}_{3}^{E}+\bar{q}_{3}^{p}=q_{3} /\left(L_{\text {ref }}^{2} U_{\text {ref }}\right)$

The dimensionless flowrates are given as

$$
\begin{aligned}
& \bar{q}_{1}^{E}=2 \int_{-\bar{h}-\bar{h}_{1}}^{-\bar{h}} \int_{0}^{\bar{w}} \bar{u}_{1}^{E}(\bar{y}, \bar{z}) d \bar{z} d \bar{y} \\
& \bar{q}_{1}^{p}=2 \int_{-\bar{h}-\bar{h}_{1}}^{-\bar{h}} \int_{0}^{\bar{w}} \bar{u}_{1}^{p}(\bar{y}, \bar{z}) d \bar{z} d \bar{y} \\
& \bar{q}_{2}^{E}=2 \int_{-\bar{h}}^{\bar{h}} \int_{0}^{\bar{w}} \bar{u}_{2}^{E}(\bar{y}, \bar{z}) d \bar{z} d \bar{y} \\
& \bar{q}_{2}^{p}=2 \int_{-\bar{h}}^{\bar{h}} \int_{0}^{\bar{w}} \bar{u}_{2}^{p}(\bar{y}, \bar{z}) d \bar{z} d \bar{y} \\
& \text { and } \\
& \bar{q}_{3}^{E}=2 \int_{\bar{h}}^{\bar{h}+\bar{h}_{3}} \int_{0}^{\bar{w}} \bar{u}_{3}^{E}(\bar{y}, \bar{z}) d \bar{z} d \bar{y} \\
& \bar{q}_{3}^{p}=2 \int_{\bar{h}}^{\bar{h}+\bar{h}_{3}} \int_{0}^{\bar{w}} \bar{u}_{3}^{p}(\bar{y}, \bar{z}) d \bar{z} d \bar{y}
\end{aligned}
$$

Substituting $\bar{u}_{1}^{E}, \bar{u}_{1}^{p}, \bar{u}_{2}^{E}, \bar{u}_{2}^{p}, \bar{u}_{3}^{E}$ and $\bar{u}_{3}^{p}$ into Eqs. (52)-(57), respectively, yields the dimensionless volumetric flowrates as 


$$
\begin{aligned}
& \bar{q}_{1}^{E}=\sqrt{\frac{2}{\bar{w}}} \sum_{j=0}^{\infty} \frac{2 \sin \left(\lambda_{j} \bar{w}\right)}{\lambda_{j}}\left\{\frac{1}{\lambda_{j}} \tanh \left[\lambda_{j}\left(\bar{h}+\bar{h}_{1}\right)\right] \times\left[\sinh \left(\lambda_{j}\left(\bar{h}+\bar{h}_{1}\right)\right)-\sinh \left(\lambda_{j} \bar{h}\right)\right]+\frac{1}{\lambda_{j}}\left[\cosh \left(\lambda_{j} \bar{h}\right)-\cosh \left(\lambda_{j}\left(\bar{h}+\bar{h}_{1}\right)\right)\right]\right\} b_{1 j}^{E} \\
& +\sqrt{\frac{2}{\bar{w}}} \sum_{j=0}^{\infty} \frac{2 \sin \left(\lambda_{j} \bar{w}\right)}{\lambda_{j}} \times\left\{\frac{1}{\lambda_{j}} \frac{\phi_{1 j}\left(-\bar{h}-\bar{h}_{1}\right)}{\cosh \left[\lambda_{j}\left(\bar{h}+\bar{h}_{1}\right)\right]} \times\left[\sinh \left(\lambda_{j} \bar{h}\right)-\sinh \lambda_{j}\left(\bar{h}+\bar{h}_{1}\right)\right]+\phi_{1 j}^{l}(-\bar{h})-\phi_{1 j}^{l}\left(-\bar{h}-\bar{h}_{1}\right)\right\} \\
& \bar{q}_{1}^{p}=\sum_{j=0}^{\infty} \frac{4}{\bar{w}} \sin \left(\lambda_{j} \bar{w}\right) \frac{\operatorname{Re}}{\lambda_{j}^{4}} \frac{d \bar{p}}{d \bar{x}}(-1)^{j}\left\{\frac{\sinh \left(\lambda_{j}\left(\bar{h}+\bar{h}_{1}\right)-\sinh \left(\lambda_{j} \bar{h}\right)\right.}{\lambda_{j} \cosh \left[\lambda_{j}\left(\bar{h}_{1}+\bar{h}\right)\right]}-\bar{h}_{1}\right\}+\left\{\left[\cosh \left(\lambda_{j} \bar{h}\right)-\cosh \left(\lambda_{j}\left(\bar{h}+\bar{h}_{1}\right)\right]\right\} b_{1 j}^{p}\right. \\
& +\sum_{j=0}^{\infty} 2 \sqrt{\frac{2}{\bar{w}}} \frac{\sin \left(\lambda_{j} \bar{w}\right)}{\lambda_{j}^{2}}\left\{\tanh \left[\lambda_{j}\left(\bar{h}_{1}+\bar{h}\right)\right]\left[\sinh \left(\lambda_{j}\left(\bar{h}+\bar{h}_{1}\right)-\sinh \left(\lambda_{j} \bar{h}\right)\right]\right\}\right. \\
& \bar{q}_{2}^{E}=4 \sqrt{\frac{2}{\bar{w}}} \sum_{j=0}^{\infty} \frac{a_{2 j}^{E} \sinh \left(\lambda_{j} \bar{h}\right) \sin \left(\lambda_{j} \bar{w}\right)}{\lambda_{j}^{2}} \\
& \bar{q}_{2}^{p}=4 \sqrt{\frac{2}{\bar{w}}} \sum_{j=0}^{\infty}\left[a_{2 j}^{p} \sinh \left(\lambda_{j} \bar{h}\right)-\frac{(-1)^{j}}{\beta_{2} \lambda_{j}^{2}} \operatorname{Re} \times \frac{d \bar{p}}{d \bar{x}} \times \sqrt{\frac{2}{\bar{w}}} \bar{h}\right] \frac{\sin \left(\lambda_{j} \bar{w}\right)}{\lambda_{j}^{2}} \\
& \bar{q}_{3}^{E}=2 \sqrt{\frac{2}{\bar{w}}} \sum_{j=0}^{\infty}\left\{\frac{1}{\lambda_{j}}\left\{-\tanh \left[\lambda_{j}\left(\bar{h}+\bar{h}_{3}\right)\right]\left[\sinh \left(\lambda_{j}\left(\bar{h}^{\prime}+\bar{h}_{3}\right)\right)-\sinh \left(\lambda_{j} \bar{h}\right)\right]+\cosh \left(\lambda_{j}\left(\bar{h}+\bar{h}_{3}\right)\right)-\cosh \left(\lambda_{j} \bar{h}\right)\right\} b_{3 j}^{E} .\right. \\
& \left.-\frac{1}{\lambda_{j}} \frac{\phi_{3 j}\left(\bar{h}+\bar{h}_{j}\right)}{\cosh }\left[\lambda_{j}\left(\bar{h}+\bar{h}_{3}\right)\right] \times\left[\sinh \left(\lambda_{j}\left(\bar{h}+\bar{h}_{3}\right)\right)-\sinh \left(\lambda_{j} \bar{h}\right)\right]+\phi_{3 j}^{l}\left(\bar{h}+\bar{h}_{3}\right)-\phi_{3 j}^{l}(\bar{h})\right\} \frac{\sin \left(\lambda_{j} \bar{w}\right)}{\lambda_{j}} \\
& \bar{q}_{3}^{p}=2 \sqrt{\frac{2}{\bar{w}}} \sum_{j=0}^{\infty}\left\{\frac{1}{\lambda_{j}^{4}} \frac{\operatorname{Re}}{\beta_{3}} \frac{d \bar{p}}{d \bar{x}} \sqrt{\frac{2}{\bar{w}}}(-1)^{j}\left\{\frac{1}{\lambda_{j}} \frac{\sinh \left(\lambda_{j}\left(\bar{h}+\bar{h}_{3}\right)\right)-\sinh \left(\lambda_{j} \bar{h}\right)}{\cosh \left[\lambda_{j}\left(\bar{h}_{3}+\bar{h}\right)\right]}-\bar{h}_{3}\right\} \times \sin \left(\lambda_{j} \bar{w}\right)\right. \\
& \left.+2 \sqrt{\frac{2}{\bar{w}}}\left\{-\tanh \left[\lambda_{j}\left(\bar{h}_{3}+\bar{h}\right)\right]\left[\sinh \left(\lambda_{j}\left(\bar{h}+\bar{h}_{3}\right)\right)-\sinh \left(\lambda_{j} \bar{h}\right)\right]+\left[\cosh \left(\lambda_{j}\left(\bar{h}+\bar{h}_{3}\right)\right)-\cosh \left(\lambda_{j} \bar{h}\right)\right]\right\} b_{3 j}^{P}\right\} \frac{\sin \left(\lambda_{j} \bar{w}\right)}{\lambda_{i}^{2}}
\end{aligned}
$$

The detailed mathematical derivation of the coefficients is presented in Appendix B.

\section{Results and discussion}

In Section 2, the analytical solutions of three fluids driven by electro-osmosis and pressure gradient are obtained. In the three liquids, the two conducting fluids hold the upper and bottom parts, and the non conducting fluid holds the middle part of the rectangular channel. Many methods for determining the zeta potentials at the wall and at the interface were proposed previously [15]. The zeta potentials at the channel walls, $\xi_{1}, \xi_{2}, \xi_{4}$ and $\xi_{5}$ depend on the material properties of the wall and the ionic properties of the fluid [5]. The zeta potential between two immiscible liquids does not only depend on the ionic properties of two fluids, but also on the $\mathrm{pH}$ and the concentration of the electrolyte $[21,22]$.

\subsection{EDL potential in conducting fluids}

The dimensionless parameter $\mathrm{K}$ is defined as $\mathrm{K}=\kappa \mathrm{D}_{\mathrm{h}}$ to evaluate parameters affecting EDL profiles. $1 / \kappa$ refers to the characteristic thickness of the EDL. As the Debye-Hückel parameter $\kappa=\left(2 z_{0}^{2} e^{2} n_{0} / \varepsilon_{0} \varepsilon_{r} k_{b} T\right)^{1 / 2}$ is proportional to the square root of the bulk ionic concentration $\mathrm{n}_{0}$, the 
variation of the ionic concentration will alter the EDL thickness. In this analysis, the concentration of the two conducting fluids is in the range of $10^{-6} \sim 10^{-5} \mathrm{M}$, therefore, the bulk concentration $\mathrm{n}_{0}=6.022 \times 10^{20} \sim 6.022 \times 10^{21} \mathrm{~m}^{-3}$ and the EDL dimension parameter $\mathrm{K}=87 \sim$ 275. The EDL profiles are shown in Fig. 2 where $K=87(1 / \kappa \approx 300 \mathrm{~nm})$ and $K=275(1 / \kappa \approx 97 \mathrm{~nm})$. It shows that the value of $\mathrm{K}$ controls the dimensionless EDL thickness: a larger value of $\mathrm{K}$ corresponds to a thinner EDL.

In Fig. 1(a), the three fluids, (two conducting fluids and a non conducting fluid) are introduced through a constant pressure source. When an electric field is applied across the conducting fluids, the external electric field interacts with the net charges within the double layers and creates electroosmotic forces within the bulk conducting fluids. If the applied electric field varies, such applied electroosmotic body forces will be changed accordingly. As a result, for a given pressure gradient, the velocities and flowrates of the three liquids depend on the applied electroosmotic force.

From Eq. (25), the velocity $\bar{u}$ of three-fluid can be decomposed into two parts, $\bar{u}^{p}$ and $\bar{u}^{E} . \bar{u}^{p}$ corresponds to velocity driven by pressure gradient and $\bar{u}^{E}$ corresponds to velocity driven by electroosmotic effect. With the proposed analytical model, we investigate the following cases. (1) With zero pressure gradient is applied across the microchannel, the flow is simply a three-fluid electroosmotic flow with the flow velocity $\bar{u}^{E}$. (2) With zero applied electric, the flow is simply driven by pressure difference with the flow velocity $\bar{u}^{p}$. (3) When both the pressure gradient and the electric field are applied across the microchannel, the three-fluid is driven by the combined electroosmotic force and pressure gradient with the flow velocity $\bar{u}$.

\subsection{Three-fluid electroosmotic flow}

Fig. 3(a) shows the dimensionless velocity profiles, $\bar{u}^{E}$, at the symmetric line, at zero pressure gradient, $E_{x 1}=E_{x 3}=10 \mathrm{~V} / \mathrm{cm}$, and different viscosity ratios $\beta_{2}=0.5,1,2,3$. When electric fields are applied across the conducting fluids, the conducting fluids 1 and 3 are driven by electroosmosis, which drags the non conducting fluid 2 by the hydrodynamic shear force. The flow of the threefluid is affected by viscosity ratios, the strength of the external electric fields and electroosmotic characteristics of the conducting fluids. By this way, the non conducting fluid can be delivered by electroosmosis. 
In Section 2, general equations were derived for the EDL distribution in the conducting fluid and velocity profiles for the three-fluid electroosmotic flow through a rectangular microchannel. In the analysis, the reference potential and the applied electrical potential are taken at $25{ }^{\circ} \mathrm{C}$ as $\xi_{\text {ref }}=k_{b} T /\left(z_{0} e\right) \approx 25.7 \mathrm{mV}$ and $E_{x 1}=E_{x 3}=10 \mathrm{~V} / \mathrm{cm}$. The viscosity and density of the KCL solution is

$\mu_{\text {ref }}=10^{-3} \mathrm{~Pa}$ 's and $\rho_{\text {ref }}=10^{3} \mathrm{~kg} / \mathrm{m}^{3}$, respectively. With these reference potential and viscosity, the Helmholtz-Smoluchowski electroosmotic velocity is chosen as the reference velocity $U_{\text {ref }}=\frac{E_{x} \varepsilon \xi_{r e f}}{\mu_{\text {ref }}}=5.3 \times 10^{-5} \mathrm{~m} / \mathrm{s}$ The corresponding Reynolds number is $\operatorname{Re} \approx 0.0021$.

The flow characteristics depend on the coupling effects of the three fluids which involve the electrokinetic driving force in the conducting fluids and the interfacial phenomenon. The interfacial phenomenon is obtained from the balance of the modified stress force as shown in Eqs. (21), (22), which involves the opposite electrostatic force exerted on the interface and the hydrodynamic shear stress at the liquid-liquid interface. The velocity at the liquid-liquid interface must match, i.e. the conducting fluid and the non conducting velocities must be the same and the forces must be balanced at the interface. To investigate the effect of viscosity ratio between the three fluids, the value of $\beta_{3}=1$, whereas, $\beta_{2}$ are chosen to have different values.

The electrical body force is resulted from the interaction of the applied electric fields and the net charge density. This driving force exists only within the non-neutral charge region- the electrical double layer (EDL) in the conducting fluids 1 and 3. Liquid outside the EDL regions is set in motion passively due to the frictional stresses originating from the liquid viscosity. The velocity profile of the non conducting fluid 2 is passive. It is purely due to the interfacial shear stresses dragged by the conducting fluids on the non conducting fluid. The results indicate that the velocity profiles of the conducting fluids are strongly dependent on the viscosity ratio, $\beta$. Because the viscosity ratio is small, the flow resistance of the non conducting fluid is also small. Thus, the non conducting fluid can be driven with less flow resistance as shown in Fig. 3(a). When the viscosity ratio is higher, the flow resistance of the non conducting fluid is higher, resulting in a steeper velocity gradient at the interface of the conducting fluids.

\subsection{Three-fluid flow driven by electroosmosis and pressure gradient}


From Fig. 4, when both pressure gradient and electric field are applied, the three liquids are driven by electroosmotic body force and pressure gradient. For a given pressure gradient, the velocities and flowrates of the three liquids depend only on the applied electroosmotic force.

\subsubsection{Effect of the applied electric field on velocity profile}

Figs. 4-7 show the dimensionless velocity profiles at the symmetric line of the three fluids driven by the combined electroosmosis and pressure gradient. In the analysis, $\alpha_{1}=\alpha_{3}=0.29, \alpha_{2}=$ 0.42 and equal applied electric fields $E_{x 1}=E_{x 3}$ are specified.

The effect of applied electric fields on the dimensionless velocity profile is shown in Fig. 4. The results indicate that for a given pressure gradient, the velocity profile $\bar{u}$ of the three-fluid strongly depends on the applied electroosmotic body force; hence the volumetric flowrates of the three-fluid can be controlled. With zero electric field, $\mathrm{E}_{\mathrm{x} 1}=\mathrm{E}_{\mathrm{x} 3}=0 \mathrm{~V} / \mathrm{cm}$ the flow is in fact a pressure driven flow of a single liquid, showing the parabolic velocity profile, $\bar{u}^{p}$. We can compare the analytical solution of the three-fluid model with the one-dimensional, fully developed Navier-Stokes equation under the steady state condition [23] it is clearly seen that the results from the two different models are identical.

The relationship between $\bar{u}^{p}, \bar{u}^{E}$ and $\bar{u}$ for $d \bar{p} / d \bar{z}=1500, E_{x 1}=E_{x 3}=150 \mathrm{~V} / \mathrm{cm}$ and $\beta_{3}=\beta_{2}=1$ is

shown in Fig. 5. The result shows that the velocity profile, $\bar{u}$ of a three-fluid driven by the combined electro-osmosis and pressure gradient is the superposition of the solutions of a threefluid electroosmotic velocity profile $\bar{u}^{E}$ and a pressure-driven parabolic profile, $\bar{u}^{p}$.

Fig. 6 shows the dimensionless velocity profiles $\bar{u}_{\text {at }}$ the symmetric line when $\beta_{2}=5, \beta_{1}=\beta_{3}=$ $1, d \bar{p} / d \bar{z}=1000$. The result indicate that the velocity profile of the conducting fluid are strongly dependent on the viscosity ratio, $\beta_{2}$. For a given pressure gradient, a higher viscosity ratio $\beta_{2}$ and consequently a higher flow resistance of the non conducting fluid result in a steeper velocity gradient at the interface of the conducting fluids.

3.3.2. Effect of pressure gradient, $d \bar{p} / d \bar{z}$, on velocity profile 
Fig. 7 shows the effect of applied pressure gradient on the dimen-sionless velocity profile at the symmetric line of the three-fluid driven by the combined electroosmosis and pressure gradient. In the analysis, $\alpha_{1}=\alpha_{3}=0.29, \alpha 2=0.42, \beta_{2}=5, \beta_{1}=\beta_{3}=1$ and equal applied electric fields $E_{\mathrm{x} 1}=\mathrm{E}_{\mathrm{x} 3}=10 \mathrm{~V} / \mathrm{cm}$ are specified. The results indicate that for a given applied electric field the velocity profile $\mathrm{u}$ of the three-fluid strongly depends on the applied pressure gradient.

With zero pressure gradient ( $d \bar{p} / d \bar{z}=0$ ), the flow is simply a three-fluid electroosmotic flow $\bar{u}^{E}$. The velocity $\mathrm{u}$ increases with the applied pressure gradient as shown by the linear combination of the electroosmotic profile $\bar{u}^{E}$, and the pressure-driven profile up. At a high pressure gradient, the electroosmotic flow effect becomes weaker.

\subsubsection{Effect of viscosity ratio, $\beta_{2}$, on velocity profile}

The flow characteristics depend on the coupling effect of the three fluids which involve the electrokinetic phenomenon of the conducting fluids and the interfacial stresses at the interface of adjacent fluids. To investigate the effect of viscosity ratio between the three fluids, the value of $\beta_{3}=1$ is set to be a constant, whereas, $\beta_{2}$ are chosen to vary. Fig. 3(b) shows the dimensionless velocity profiles at the symmetric line at $d \bar{p} / d \bar{z}=1000, \mathrm{E}_{\mathrm{x} 1}=\mathrm{E}_{\mathrm{x} 3}=10 \mathrm{~V} / \mathrm{cm}$, and different viscosity ratios $\beta_{2}$ of $0.5,1,2$ and 3 respectively. The velocity profiles of the three-fluid strongly depend on the viscosity ratio, $\beta_{2}$. For a given pressure gradient and applied electric fields, if the viscosity ratio $\beta_{2}$ decreases, the non conducting fluid can be driven with less flow resistance.

The comparison between theoretical analysis and the published two-fluid experimental data [24] is shown in Fig. 8. To simulate the flow, a infinite viscosity of $\bar{\mu}_{3}$, is assumed which make the conducting fluid 3 resemble that of the channel wall. Hence we can compare our theoretical analysis with the two-fluid data. Our results agree well with the published experimental data.

Fig. 9 shows the influence of the viscosity ratio $\beta_{2}$ on the volumetric flowrate. For a given pressure gradient and the applied electric fields, volumetric flowrates of the three fluids increase with decreasing viscosity ratio $\beta_{2}$. A rapid increase in the flowrate occurs when the viscosity ratio $\beta_{2}$ decrease from 10 to 0.1 . 


\section{Conclusions}

In this paper, we presented a theoretical model of the pressure driven three-fluid flow in rectangular microchannels with electroosmotic effect. Under the Debye-Hückel linear approximation, analytical solutions of electric distribution were obtained by solving the linear Poisson-Boltzmann equation. The solutions of modified Navier-Stokes equations were presented for a steady, fully developed laminar three-fluid flow under the combined effects of pressure gradient, electro-osmosis and surface charges at the liquid-liquid interface. The comparison between the predictions of the velocity profile from the theoretical analysis and the published data show good agreement.

\section{Acknowledgement}

Haiwang Li gratefully acknowledges the Ph.D. of Engineering scholarship from Nanyang Technological University. 


\section{Appendix A}

In the following, we will define several auxiliary functions which facilitate the analytical evaluation of the pertinent expressions in the present work. All these functions are obtained through integrating matching conditions, velocity profiles and shear stress at the interface. They are defined as follow:

$$
\begin{aligned}
\phi_{1 j}(\bar{y})= & \sqrt{\bar{w}} \frac{4 \times \operatorname{Re} \times G_{x 1} \times(-1)^{j+1}}{(2 j+1) \pi \sinh \left[B_{j} \bar{h}_{1}\right]} \\
& \times\left\{\bar{\xi}_{1} \frac{\sinh \left[B_{j}(\bar{h}+\bar{y})\right]}{\left(B_{j}^{2}-\lambda_{j}^{2}\right)}-\bar{\xi}_{3} \frac{\sinh \left[B_{j}\left(\bar{h}_{1}+\bar{h}+\bar{y}\right)\right]}{\left(B_{j}^{2}-\lambda_{j}^{2}\right)}\right\} \\
& +\sqrt{\frac{2}{\bar{w}} \sum_{p=1}^{\infty} \frac{2 \times \operatorname{Re} \times G_{x 1} \times \bar{\xi}_{2} \times \lambda_{j}}{p \pi\left(A_{p 1}^{2}+\lambda_{j}^{2}\right)}\left[(-1)^{p}-1\right]} \\
& \times(-1)^{j+1} \frac{\sin \left[\frac{p \pi}{h_{1}}(\bar{h}+\bar{y})\right]}{\left[\left(\frac{p \pi}{h_{1}}\right)^{2}+\lambda_{j}^{2}\right]} \\
\phi_{3 j}(\bar{y})= & -\sqrt{\frac{\bar{w}}{2} \sum_{j=1}^{\infty} \frac{4 \times G_{3 x} \times \frac{\operatorname{Re}}{\beta_{3}}(-1)^{j+1}}{(2 j+1) \pi \sinh \left[B_{j} \bar{h}_{3}\right]\left(B_{j}^{2}-\lambda_{j}^{2}\right)}} \\
& \times\left\{\bar{\xi}_{4} \sinh \left[B_{j}(\bar{y}-\bar{h})\right]-\bar{\xi}_{6} \sinh \left[B_{j}\left(\bar{y}-\bar{h}_{3}-\bar{h}\right)\right]\right\} \\
& -\sqrt{\frac{2}{\bar{w}} \sum_{p=1}^{\infty} \frac{2 \bar{\xi}_{5} \times G_{3 x} \times \frac{R e}{\beta_{3}} \times \lambda_{j}\left[(-1)^{p}-1\right] \times(-1)^{j+1}}{p \pi}} \\
& \times \frac{\sin \left[\frac{p}{h_{3}} \pi(\bar{y}-\bar{h})\right]}{\left(A_{p 3}^{2}+\lambda_{j}^{2}\right)\left[\left(\frac{p \pi}{h_{3}}\right)^{2}+\lambda_{j}^{2}\right]}
\end{aligned}
$$




\section{Appendix B}

$$
\begin{aligned}
& A= \tanh \left[\lambda_{j}\left(\bar{h}+\bar{h}_{1}\right)\right] \cosh \left(\lambda_{j} \bar{h}\right) \sinh \left(\lambda_{j} \bar{h}\right) \\
&-\sinh \left(\lambda_{j} \bar{h}\right) \sinh \left(\lambda_{j} \bar{h}\right) \\
&-\frac{\cosh \left(\lambda_{j} \bar{h}\right)}{\beta_{2}} \tanh \left[\lambda_{j}\left(\bar{h}+\bar{h}_{1}\right)\right] \sinh \left(\lambda_{j} \bar{h}\right) \\
&+\frac{\cosh \left(\lambda_{j} \bar{h}\right) \cosh \left(\lambda_{j} \bar{h}\right)}{\beta_{2}} \\
& B=-\frac{\cosh \left(\lambda_{j} \bar{h}\right) \tanh \left[\lambda_{j}\left(\bar{h}+\bar{h}_{3}\right)\right] \sinh \left(\lambda_{j} \bar{h}\right)}{\beta_{3}} \\
&+\frac{\cosh \left(\lambda_{j} \bar{h}\right) \cosh \left(\lambda_{j} \bar{h}\right)}{\beta_{3}} \\
&+\tanh \left[\lambda_{j}\left(\bar{h}+\bar{h}_{3}\right)\right] \cosh \left(\lambda_{j} \bar{h}\right) \sinh \left(\lambda_{j} \bar{h}\right) \\
&-\sinh \left(\lambda_{j} \bar{h}\right) \sinh \left(\lambda_{j} \bar{h}\right) \\
& C=-\frac{\sinh \left(\lambda_{j} \bar{h}_{3}\right)}{2 \cosh \left[\lambda_{j}\left(\bar{h}+\bar{h}_{3}\right)\right] \sinh \left(\lambda_{j} \bar{h}\right)} \\
& D=-\frac{\sinh \left(\lambda_{j} \bar{h}_{1}\right)}{2 \cosh \left[\lambda_{j}\left(\bar{h}+\bar{h}_{1}\right)\right] \sinh \left(\lambda_{j} \bar{h}\right)} \\
& G=-\frac{\phi_{3 j}\left(\bar{h}+\bar{h}_{3}\right) \cosh \left(\lambda_{3 j} \bar{h}\right)}{2 \sinh \left(\lambda_{j} \bar{h}\right) \cosh \left[\lambda_{j}\left(\bar{h}+\bar{h}_{3}\right)\right]}+\frac{\phi_{3 j}(\bar{h})}{2 \sinh \left(\lambda_{j} \bar{h}\right)} \\
&+\frac{\phi_{1 j}\left(-\bar{h}-\bar{h}_{1}\right) \cosh \left(\lambda_{j} \bar{h}\right)}{2 \sinh \left(\lambda_{j} \bar{h}\right) \cosh \left[\lambda_{j}\left(\bar{h}+\bar{h}_{1}\right)\right]}-\frac{\phi_{1 j}(-\bar{h})}{2 \sinh \left(\lambda_{j} \bar{h}\right)} \\
& H= \frac{\sinh \left(\lambda_{j} \bar{h}\right)}{A}\left\{\frac{\phi_{1 j}\left(-\bar{h}-\bar{h}_{1}\right)}{\cosh \left[\lambda_{j}\left(\bar{h}+\bar{h}_{1}\right)\right]} \cosh \left(\lambda_{j} \bar{h}\right)-\phi_{1 j}(-\bar{h})\right\} \\
&-\frac{\cosh \left(\lambda_{j} \bar{h}\right)}{A \beta_{2}}\left\{\frac{\phi_{1 j}^{\prime}(-\bar{h})}{\lambda_{j}}+\frac{M_{1} \bar{\rho}_{q 1}^{s}(\bar{z})}{\lambda_{j}}+\frac{\phi_{1 j}\left(-\bar{h}-\bar{h}_{1}\right)}{\cosh \left[\lambda_{j}\left(\bar{h}+\bar{h}_{1}\right)\right]} \sinh \left(\lambda_{j} \bar{h}\right)\right\} \\
& \hline
\end{aligned}
$$




$$
\begin{aligned}
& I=\frac{\cosh \left(\lambda_{j} \bar{h}\right) \sinh \left(\lambda_{j} \bar{h}\right) \phi_{j}\left(\bar{h}+\bar{h}_{3}\right)}{B \beta_{3} \cosh \left[\lambda_{j}\left(\bar{h}+\bar{h}_{3}\right)\right]}-\frac{\cosh \left(\lambda_{j} \bar{h}\right)}{B \beta_{3} \lambda_{j}} \phi_{3 j}^{\prime}(\bar{h}) \\
& +\frac{\phi_{3 j}(\bar{h}) \sinh \left(\lambda_{j} \bar{h}\right)}{B}-\frac{\cosh \left(\lambda_{j} \bar{h}\right) M_{3} \bar{\rho}_{q 3}^{s}(\bar{z})}{B \beta_{3} \lambda_{j}} \\
& -\frac{\phi_{3 j}\left(\bar{h}+\bar{h}_{3}\right)}{B \cosh \left[\lambda_{j}\left(\bar{h}+\bar{h}_{3}\right)\right]} \cosh \left(\lambda_{j} \bar{h}\right) \sinh \left(\lambda_{j} \bar{h}\right) \\
& J=\frac{1}{2}\left\{\tanh \left[\lambda_{j}\left(\bar{h}+\bar{h}_{1}\right)\right]-\tanh \left(\lambda_{j} \bar{h}\right)\right\} \\
& \phi_{1 j}^{\prime}(\bar{y})=\sqrt{\frac{\bar{w}}{2}} \frac{4 \times \operatorname{Re} \times G_{x 1} \times B_{j} \times(-1)^{j+1}}{(2 j+1) \pi \sinh \left[B_{j} h_{1}\right]} \\
& \times\left\{\bar{\xi}_{1} \frac{\cosh \left[B_{j}(\bar{h}+\bar{y})\right]}{\left(B_{j}^{2}-\lambda_{j}^{2}\right)}-\bar{\xi}_{3} \frac{\cosh \left[B_{j}\left(h_{1}+\bar{h}+\bar{y}\right)\right]}{\left(B_{j}^{2}-\lambda_{j}^{2}\right)}\right\} \\
& +\sqrt{\frac{2}{\bar{w}}} \sum_{p=1}^{\infty} \frac{2 \times \operatorname{Re} \times G_{x 1} \times \bar{\xi}_{2} \times \lambda_{j}}{h_{1}\left(A_{p 1}^{2}+\lambda_{j}^{2}\right)}\left[(-1)^{p}-1\right] \\
& \times(-1)^{j+1} \frac{\cos \left[\frac{p \pi}{h_{1}}(\bar{h}+\bar{y})\right]}{\left[\left(\frac{p \pi}{h_{1}}\right)^{2}+\lambda_{j}^{2}\right]} \\
& \phi_{3 j}^{\prime}(\bar{y})=-\sqrt{\frac{\bar{w}}{2}} \sum_{j=1}^{\infty} \frac{4 \times G_{3 x} \times B_{j} \times \frac{\mathrm{Re}}{\beta_{3}}(-1)^{j+1}}{(2 j+1) \pi \sinh \left[B_{j} \bar{h}_{3}\right]\left(B_{j}^{2}-\lambda_{j}^{2}\right)} \times\left\{\bar{\xi}_{4} \cosh \left[B_{j}(\bar{y}-\bar{h})\right]\right. \\
& \left.-\bar{\xi}_{6} \cosh \left[B_{j}\left(\bar{y}-\bar{h}_{3}-\bar{h}\right)\right]\right\} \\
& -\sqrt{\frac{2}{\bar{w}}} \sum_{p=1}^{\infty} \frac{2 \bar{\xi}_{5} \times G_{3 x} \times \frac{\mathrm{Re}}{\beta_{3}} \times \lambda_{j}\left[(-1)^{p}-1\right] \times(-1)^{j+1}}{\bar{h}_{3}} \\
& \times \frac{\cos \left[\frac{p}{h_{3}} \pi(\bar{y}-\bar{h})\right]}{\left(A_{p 3}^{2}+\lambda_{j}^{2}\right)\left[\left(\frac{p \pi}{h_{3}}\right)^{2}+\lambda_{j}^{2}\right]}
\end{aligned}
$$




$$
\begin{aligned}
& M=\frac{1}{\lambda_{j}^{3}} \operatorname{Re} \frac{d \bar{p}}{d \bar{x}} \sqrt{\frac{2}{\bar{w}}} \times(-1)^{j} \\
& \times\left\{\frac{1}{2 \beta_{3} \cosh \left[\lambda_{j}\left(\bar{h}_{3}+\bar{h}\right)\right]}-\frac{1}{2 \cosh \left(\lambda_{j} \bar{h}\right) \beta_{3}}+\frac{1}{2 \cosh \left[\lambda_{j}\left(\bar{h}_{1}+\bar{h}\right)\right]}-\frac{1}{2 \cosh \left(\lambda_{j} \bar{h}\right)}\right\} \\
& +\frac{(-1)^{j}}{\cosh \left(\lambda_{j} \bar{h}\right) \beta_{2} \lambda_{j}^{3}} \operatorname{Re} \times \frac{d \bar{p}}{d \bar{x}} \times \sqrt{\frac{2}{\bar{w}}} \\
& N=-\frac{\sinh \left(\lambda_{j} \bar{h}_{3}\right)}{2 \cosh \left(\lambda_{j} \bar{h}\right) \cosh \left[\lambda_{j}\left(\bar{h}_{3}+\bar{h}\right)\right]} \\
& Q=\frac{\sinh \left(\lambda_{j} \bar{h}_{1}\right)}{2 \cosh \left(\lambda_{j} \bar{h}\right) \cosh \left[\lambda_{j}\left(\bar{h}_{1}+\bar{h}\right)\right]} \\
& O=\frac{1}{\lambda_{j}^{3}} \operatorname{Re} \frac{d \bar{p}}{d \bar{x}} \sqrt{\frac{2}{\bar{w}}}(-1)^{j} \\
& \times\left\{\frac{\operatorname{coth}\left(\lambda_{j} \bar{h}\right)}{2 \beta_{3} \cosh \left[\lambda_{j}\left(\bar{h}_{3}+\bar{h}\right)\right]}-\frac{1}{2 \sinh \left(\lambda_{j} \bar{h}\right) \beta_{3}}-\frac{\operatorname{coth}\left(\lambda_{j} \bar{h}\right)}{2 \cosh \left[\lambda_{j}\left(\bar{h}_{1}+\bar{h}\right)\right]}+\frac{1}{2 \sinh \left(\lambda_{j} \bar{h}\right)}\right\} \\
& P=-\frac{\sinh \left(\lambda_{j} \bar{h}_{3}\right)}{2 \sinh \left(\lambda_{j} \bar{h}\right) \cosh \left[\lambda_{j}\left(\bar{h}_{3}+\bar{h}\right)\right]} \\
& R=-\frac{\sinh \left(\lambda_{j} \bar{h}_{1}\right)}{2 \sinh \left(\lambda_{j} \bar{h}\right) \cosh \left[\lambda_{j}\left(\bar{h}_{1}+\bar{h}\right)\right]} \\
& S=\frac{\sinh \left(\lambda_{j} \bar{h}\right) \sinh \left(\lambda_{j} \bar{h}_{1}\right)}{\cosh \left[\lambda_{j}\left(\bar{h}_{1}+\bar{h}\right)\right]}+\frac{\cosh \left(\lambda_{j} \bar{h}\right) \cosh \left(\lambda_{j} \bar{h}_{1}\right)}{\beta_{2} \cosh \left[\lambda_{j}\left(\bar{h}_{1}+\bar{h}\right)\right]} \\
& T=\frac{(-1)^{j} \sinh \left(\lambda_{j} \bar{h}\right)}{\beta_{2} \lambda_{j}^{3}} \operatorname{Re} \times \frac{d \bar{p}}{d \bar{x}} \times \sqrt{\frac{2}{\bar{w}}}+\frac{1}{\lambda_{j}^{3}} \operatorname{Re} \frac{d \bar{p}}{d \bar{x}} \sqrt{\frac{2}{\bar{w}}}(-1)^{j} \\
& \times\left\{\frac{\sinh \left(\lambda_{j} \bar{h}\right) \cosh \left(\lambda_{j} \bar{h}\right)}{\cosh \left[\lambda_{j}\left(\bar{h}_{1}+\bar{h}\right)\right]}-\sinh \left(\lambda_{j} \bar{h}\right)-\frac{\cosh \left(\lambda_{j} \bar{h}\right) \sinh \left(\lambda_{j} \bar{h}\right)}{\beta_{2} \cosh \left[\lambda_{j}\left(\bar{h}_{1}+\bar{h}\right)\right]}\right\} \\
& U=\frac{(-1)^{j} \sinh \left(\lambda_{j} \bar{h}\right)}{\beta_{2} \lambda_{j}^{3}} \operatorname{Re} \\
& \times \frac{d \bar{p}}{d \bar{x}} \times \sqrt{\frac{2}{\bar{w}}}+\frac{1}{\lambda_{j}^{3}} \frac{\operatorname{Re}}{\beta_{3}} \frac{d \bar{p}}{d \bar{x}} \sqrt{\frac{2}{\bar{w}}}(-1)^{j} \\
& \times\left\{-\frac{\cosh \left(\lambda_{j} \bar{h}\right) \sinh \left(\lambda_{j} \bar{h}\right)}{\left(\beta_{2} / \beta_{3}\right) \cosh \left[\lambda_{j}\left(\bar{h}_{3}+\bar{h}\right)\right]}+\frac{\sinh \left(\lambda_{j} \bar{h}\right) \cosh \left(\lambda_{j} \bar{h}\right)}{\cosh \left[\lambda_{j}\left(\bar{h}_{3}+\bar{h}\right)\right]}-\sinh \left(\lambda_{j} \bar{h}\right)\right\} \\
& V=\frac{\cosh \left(\lambda_{j} \bar{h}\right) \cosh \left(\lambda_{j} \bar{h}\right)}{\left(\beta_{2} / \beta_{3}\right) \cosh \left[\lambda_{j}\left(\bar{h}_{3}+\bar{h}\right)\right]}+\frac{\sinh \left(\lambda_{j} \bar{h}\right) \sinh \left(\lambda_{j} \bar{h}_{3}\right)}{\cosh \left[\lambda_{j}\left(\bar{h}_{3}+\bar{h}\right)\right]}
\end{aligned}
$$

where the parameter functions $\bar{\phi}_{1 j}^{I}, \bar{\phi}_{3 j}^{I}$ are defined as

$$
\phi_{1 j}^{l}(\bar{y})=\sqrt{\frac{\bar{w}}{2}} \frac{4 \times \operatorname{Re} \times G_{x 1} \times(-1)^{j+1}}{(2 j+1) \pi \times B_{j} \times \sinh \left[B_{j} \bar{h}_{1}\right]}
$$




$$
\begin{aligned}
& \times\left\{\bar{\xi}_{1} \frac{\cosh \left[B_{j}(\bar{h}+\bar{y})\right]}{\left(B_{j}^{2}-\lambda_{j}^{2}\right)}-\bar{\xi}_{3} \frac{\cosh \left[B_{j}\left(\bar{h}_{1}+\bar{h}+\bar{y}\right)\right]}{\left(B_{j}^{2}-\lambda_{j}^{2}\right)}\right\} \\
& -\sqrt{\frac{2}{\bar{w}}} \sum_{p=1}^{\infty} \frac{2 \times \operatorname{Re} \times G_{x 1} \times \bar{\xi}_{2} \times \lambda_{j} \times \bar{h}_{1}}{(p \pi)^{2}\left(A_{p 1}^{2}+\lambda_{j}^{2}\right)}\left[(-1)^{p}-1\right] \\
& \times(-1)^{j+1} \frac{\cos \left[\frac{p \pi}{h_{1}}(\bar{h}+\bar{y})\right]}{\left[\left(\frac{p \pi}{h_{1}}\right)^{2}+\lambda_{j}^{2}\right]} \\
& b_{3 j}^{I}(\bar{y})=-\sqrt{\frac{\bar{w}}{2}} \sum_{j=1}^{\infty} \frac{4 \times G_{3 x} \times \frac{\operatorname{Re}}{\beta_{3}}(-1)^{j+1}}{(2 j+1) \pi \times B_{j} \times \sinh \left[B_{j} \bar{h}_{3}\right]\left(B_{j}^{2}-\lambda_{j}^{2}\right)} \\
& \times\left\{\bar{\xi}_{4} \cosh \left[B_{j}(\bar{y}-\bar{h})\right]-\bar{\xi}_{6} \cosh \left[B_{j}\left(\bar{y}-\bar{h}_{3}-\bar{h}\right)\right]\right\} \\
& +\sqrt{\frac{2}{\bar{w}}} \sum_{p=1}^{\infty} \\
& \times \frac{2 \bar{\xi}_{5} \times G_{3 x} \times \frac{\mathrm{Re}}{\beta_{3}} \times \bar{h}_{3} \times \lambda_{j}\left[(-1)^{p}-1\right] \times(-1)^{j+1}}{(p \pi)^{2}} \\
& \times \frac{\cos \left[\frac{p}{h_{3}} \pi(\bar{y}-\bar{h})\right]}{\left(A_{p 3}^{2}+\lambda_{j}^{2}\right)\left[\left(\frac{p \pi}{h_{3}}\right)^{2}+\lambda_{j}^{2}\right]}
\end{aligned}
$$




\section{References}

[1] T. Stiles, R. Fallon, T. Vestad, J. Oakey, D.W.M. Marr, J. Squier, R. Jimenez, Hydrodynamic focusing for vacuum-pumped microfluidics, Microfluidics and Nanofluidics 1 (2005) 280-283.

[2] P.J. Crosland-Taylor, A device for counting small particles suspended in a fluid through a tube, Nature 171 (1953) 37-38.

[3] D. Huh, Y.C. Tung, H.H. Wei, J.B. Grotberg, S.J. Skerlos, K. Kurabayashi, S. Takayama, Use of air-liquid two-phase flow in hydrophobic microfluidic channels for disposable flow cytometers, Biomedical Microdevices 4 (2002) 141-149.

[4] G.B. Lee, B.H. Hwei, G.R. Huang, Micromachined pre-focused M x N flow switches for continuous multi-sample injection, Journal of Micromechanics and Microengineering 11 (2001) 654-661.

[5] D. Erickson, D. Li, C. Werner, An improved method of determining the - potential and surface conductance, Journal of Colloid and Interface Science 232 (2000) 186-197.

[6] L.M. Fu, R.J. Yang, C.H. Lin, Y.J. Pan, G.B. Lee, Electrokinetically driven micro flow cytometers with integrated fiber optics for on-line cell/particle detection, Analytica Chimica Acta 507 (2004) 163-169.

[7] G.D. Ngoma, F. Erchiqui, Pressure gradient and electroosmotic effects on two immiscible fluids in a microchannel between two parallel plates, Journal of Micromechanics and Microengineering 16 (2006) 83-91.

[8] R.J. Yang, C.C. Chang, S.B. Huang, G.B. Lee, A new focusing model and switching approach for electrokinetic flow inside microchannels, Journal of Micromechanics and Microengineering 15 (2005) 2141-2148.

[9] J.Y. Lin, L.M. Fu, R.J. Yang, Numerical simulation of electrokinetic focusing in microfluidic chips, Journal of Micromechanics and Microengineering 12 (2002) 955-961. 
[10] L.M. Fu, R.J. Yang, G.B. Lee, Electrokinetic focusing injection methods on microfluidic devices, Analytical Chemistry 75 (2003) 1905-1910.

[11] L.M. Fu, R.J. Yang, G.B. Lee, Y.J. Pan, Multiple injection techniques for microfluidic sample handling, Electrophoresis 24 (2003) 3026-3032.

[12] Y. Gao, C. Wang, T.N. Wong, C. Yang, N.T. Nguyen, K.T. Ooi, Electro-osmotic control of the interface position of two-liquid flow through a microchannel, Journal of Micromechanics and Microengineering 17 (2007) 358-366.

[13] Y. Gao, T.N. Wong, C. Yang, K.T. Ooi, Two-fluid electroosmotic flow in microchannels, Journal of Colloid and Interface Science 284 (2005) 306-314.

[14] Z. Wu, N.T. Nguyen, Hydrodynamic focusing in microchannels under consideration of diffusive dispersion: theories and experiments, Sensors and Actuators B: Chemical 107 (2005) 965-974.

[15] R.J. Hunter, Zeta Potential in Colloid Science: Principles and Applications Harcourt Brace Jovanovich, London San Diego New York Berkely Boston Sydney Tokyo Toronto, 1981, pp. $15-75$.

[16] R.G. Cox, T.G.M. Van De Ven, Electroviscous forces on a charged particle suspended in a flowing liquid, Journal of Fluid Mechanics 338 (1997) 1-34.

[17] R. F Probstein, Physicochemical Hydrodynamics: An Introduction, John Wiley\& Sons, Inc., New York, 1994.

[18] J.C. Baygents, D.A. Saville, Electrophoresis of small particles and fluid globules in weak electrolytes, Journal of Colloid and Interface Science 146 (1991) 9-37.

[19] P. Dutta, A. Beskok, T.C. Warburton, Electroosmotic flow control in complex microgeometries, Journal of Microelectromechanical Systems 11 (2002) 36- 44.

[20] G.Y. Tang, C. Yang, J.C. Chai, H.Q. Gong, Joule heating effect on electroosmotic flow and mass species transport in a microcapillary, International Journal of Heat and Mass Transfer 47 (2004) 215-227. 
[21] Y. Gu, D. Li, An electrical suspension method for measuring the electric charge on small silicone oil droplets dispersed in aqueous solutions, Journal of Colloid and Interface Science 195 (1997) 343-352.

[22] Y. Gu, D. Li, The n-potential of silicone oil droplets dispersed in aqueous solutions, Journal of Colloid and Interface Science 206 (1998) 346-349.

[23] William M. Deen, Analysis of transport phenomena, Oxford University Press, New York, 1998 (pp. 208-248).

[24] C. Wang, Y. Gao, N.T. Nguyen, T.N. Wong, C. Yang, K.T. Ooi, Interface control of pressure-driven two-fluid flow in microchannels using electroosmosis, Journal of Micromechanics and Microengineering 15 (2005) 2289-2297. 


\section{List of Figures}

Fig. 1 Schematic representation of the three-fluid electroosmotic stratified flow. (a) Schematic of the system of three-fluid flow in microchannel. (b) Schematic of the cross section of three-fluid flow in microchannel and coordinate system.

Fig. 2 EDL profiles at the symmetric line in the two conducting fluids

Fig. 3 Dimensionless velocity distribution at the symmetric line for different values of viscosity ratio, $\beta_{2}\left(\beta_{1}=\beta_{3}=1\right)$. (a) $\left(\mathrm{E}_{\mathrm{x} 1}=\mathrm{E}_{\mathrm{x} 3}=10 \mathrm{~V} / \mathrm{cm}, d \bar{p} / d \bar{z}=0\right)$. (b) $\left(\mathrm{E}_{\mathrm{x} 1}=\mathrm{E}_{\mathrm{x} 3}\right.$ $\left.=10 \mathrm{~V} / \mathrm{cm}^{d \bar{p}} / d \bar{z}=1000\right)$.

Fig. 4 Dimensionless velocity distribution at the symmetric line for different values of electric field $\mathrm{E}_{\mathrm{x} 1}$ and $\mathrm{E}_{\mathrm{x} 3}\left(d \bar{p} / d \bar{z}=1000, \beta_{1}=1, \beta_{2}=1, \beta_{3}=1\right)$.

Fig. 5 Schematic of the relationship of $\bar{u}^{p}\left(d \bar{p} / d \bar{z}=1500, \mathrm{E}_{\mathrm{x} 1}=\mathrm{E}_{\mathrm{x} 3}=0 \mathrm{~V} / \mathrm{cm}\right), \bar{u}^{\bar{E}_{1}}(d \bar{p} / d \bar{z}=$ $\left.0, \mathrm{E}_{\mathrm{x} 1}=\mathrm{E}_{\mathrm{x} 3}=150 \mathrm{~V} / \mathrm{cm}\right)$, and $\bar{u} \quad\left(d \bar{p} / d \bar{z}=1500, \mathrm{E}_{\mathrm{x} 1}=\mathrm{E}_{\mathrm{x} 3}=150 \mathrm{~V} / \mathrm{cm}\right)$, when $\beta_{1}$ $=1, \beta_{2}=1, \beta_{3}=1$.

Fig. 6 Dimensionless velocity distribution at the symmetric line for different values of electric field $E_{\mathrm{x} 1}$ and $\mathrm{E}_{\mathrm{x} 3}\left(d \bar{p} / d \bar{z}=1000, \beta_{1}=1, \beta_{2}=5, \beta_{3}=1\right)$.

Fig. 7 Dimensionless velocity distribution at the symmetric line for different values of pressure gradient, ${ }^{d \bar{p}} / d \bar{z}\left(E_{\mathrm{x} 1}=E_{\mathrm{x} 3}=10 \mathrm{~V} / \mathrm{cm}, \beta_{1}=1, \beta_{2}=5, \beta_{3}=1\right)$.

Fig. 8 Comparison of the velocity profile between theoretical model and the two-fluid experimental data [24] $\left(\mathrm{E}_{\mathrm{x} 1}=\mathrm{E}_{\mathrm{x} 3}=-40 \mathrm{~V} / \mathrm{cm}, \beta_{1}=1, \beta_{2}=1.5, \beta_{3}=104\right)$.

Fig. 9 Dimensionless volumetric flowrates versus viscosity ratio $\beta_{2}\left(E_{x 1}=E_{x 3}=10\right.$ $\left.\mathrm{V} / \mathrm{cm}, d \bar{p} / d \bar{z}=1000, \beta_{1}=\beta_{3}=1\right)$. 


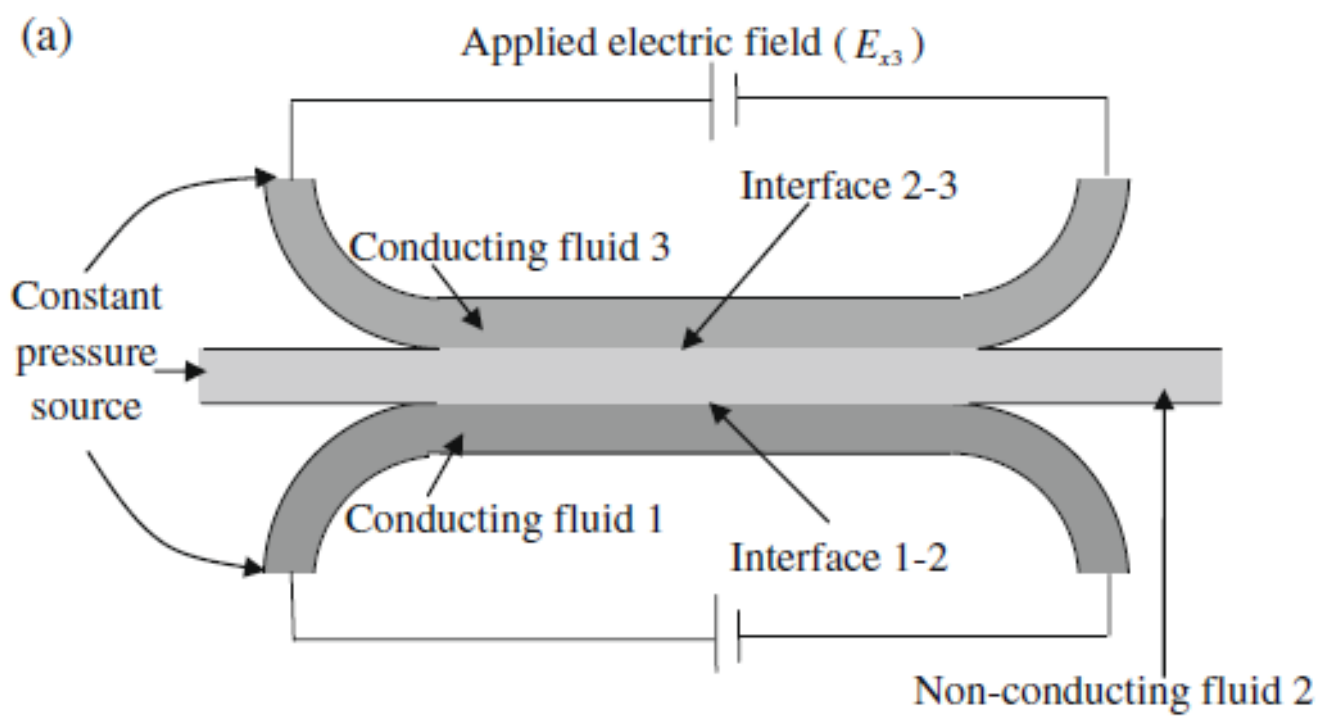

Applied electric field $\left(E_{x 1}\right)$

(b)

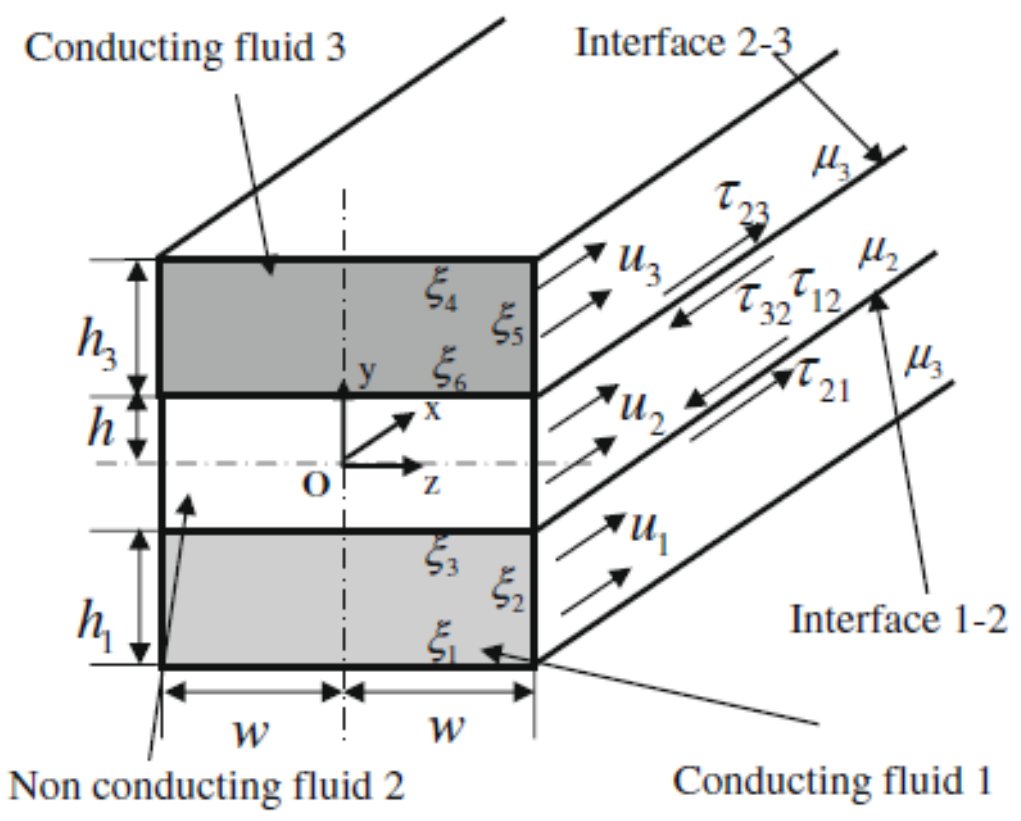

Fig. 1 


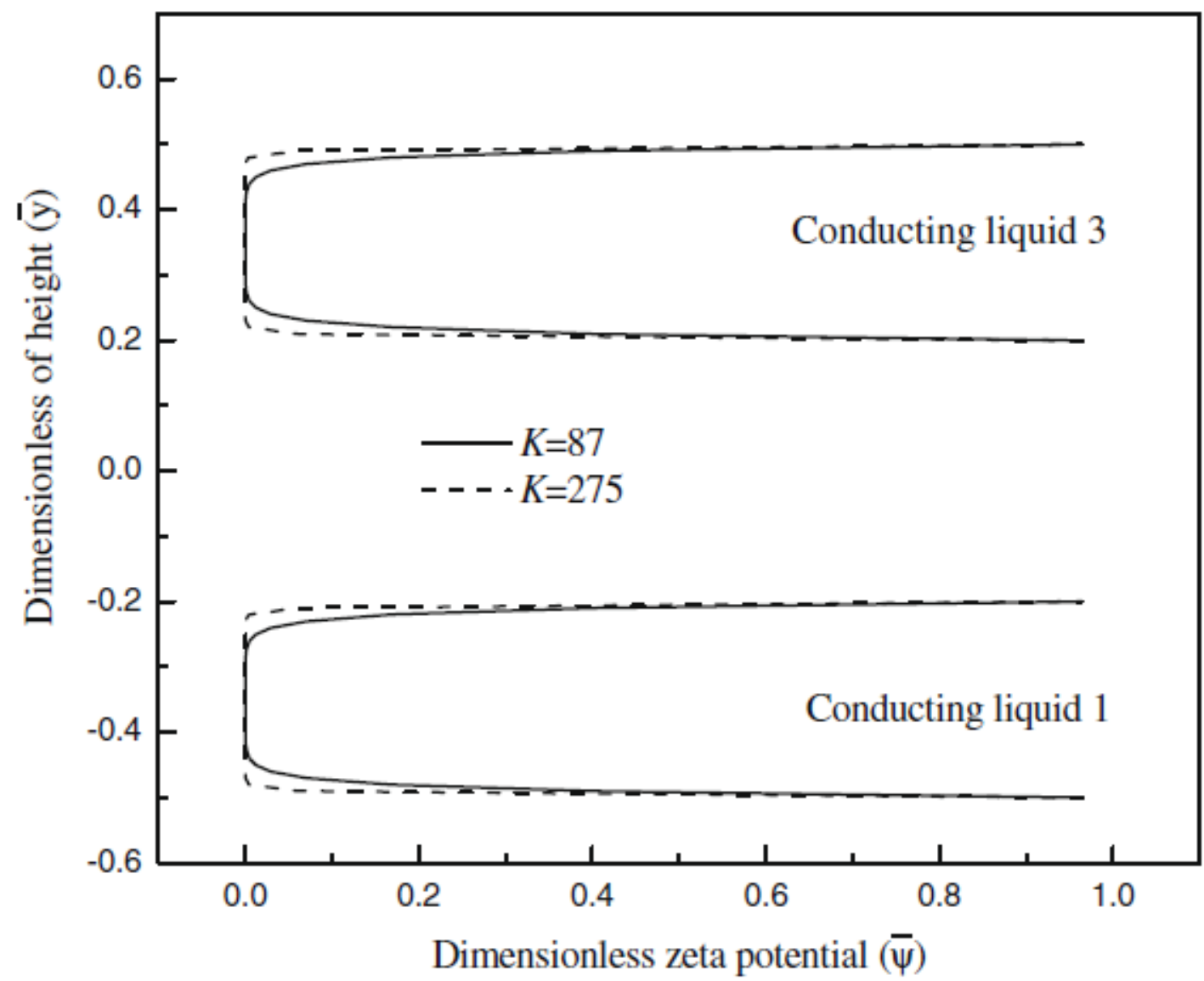

Fig. 2 

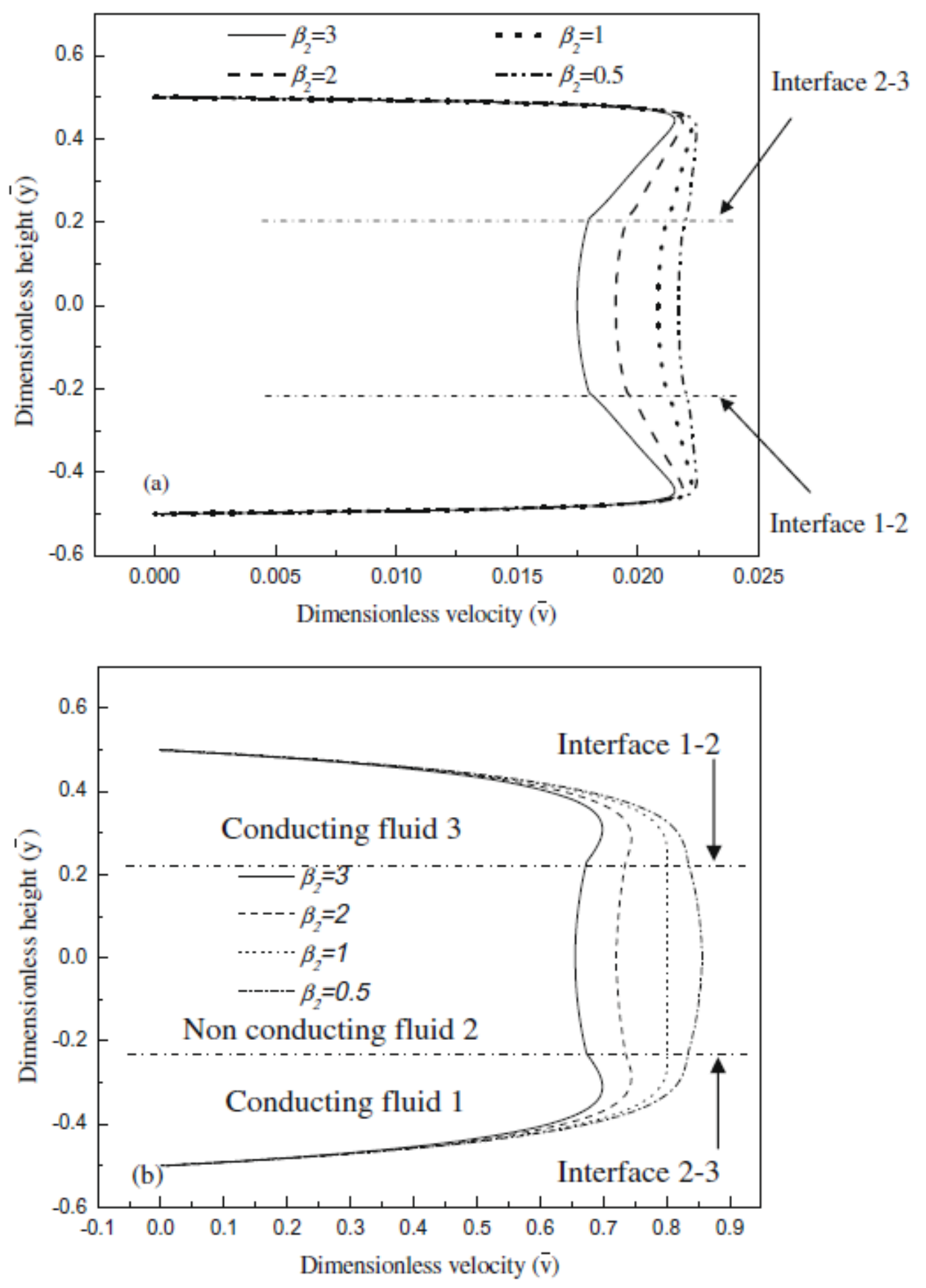

Fig. 3 


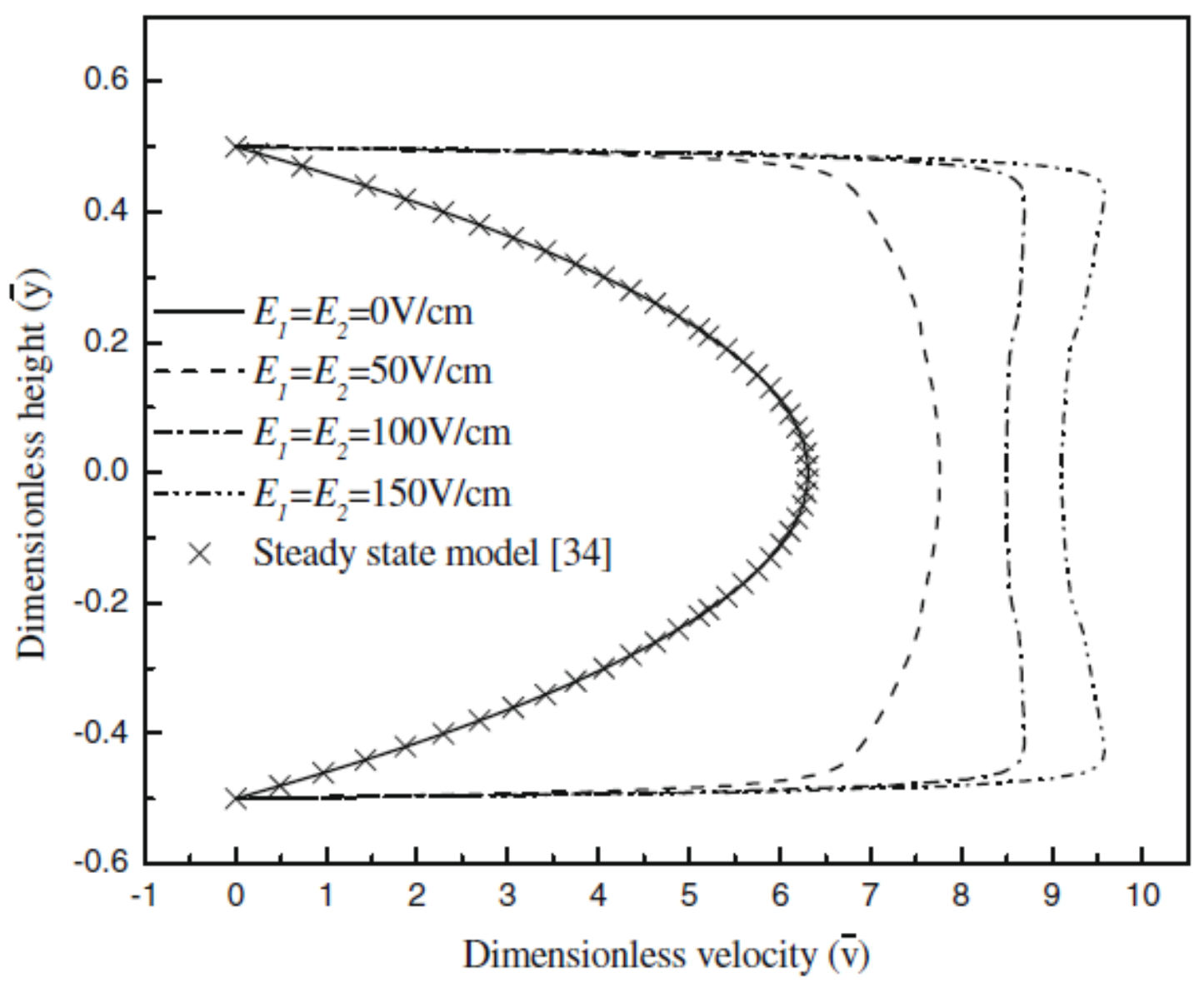

Fig. 4 


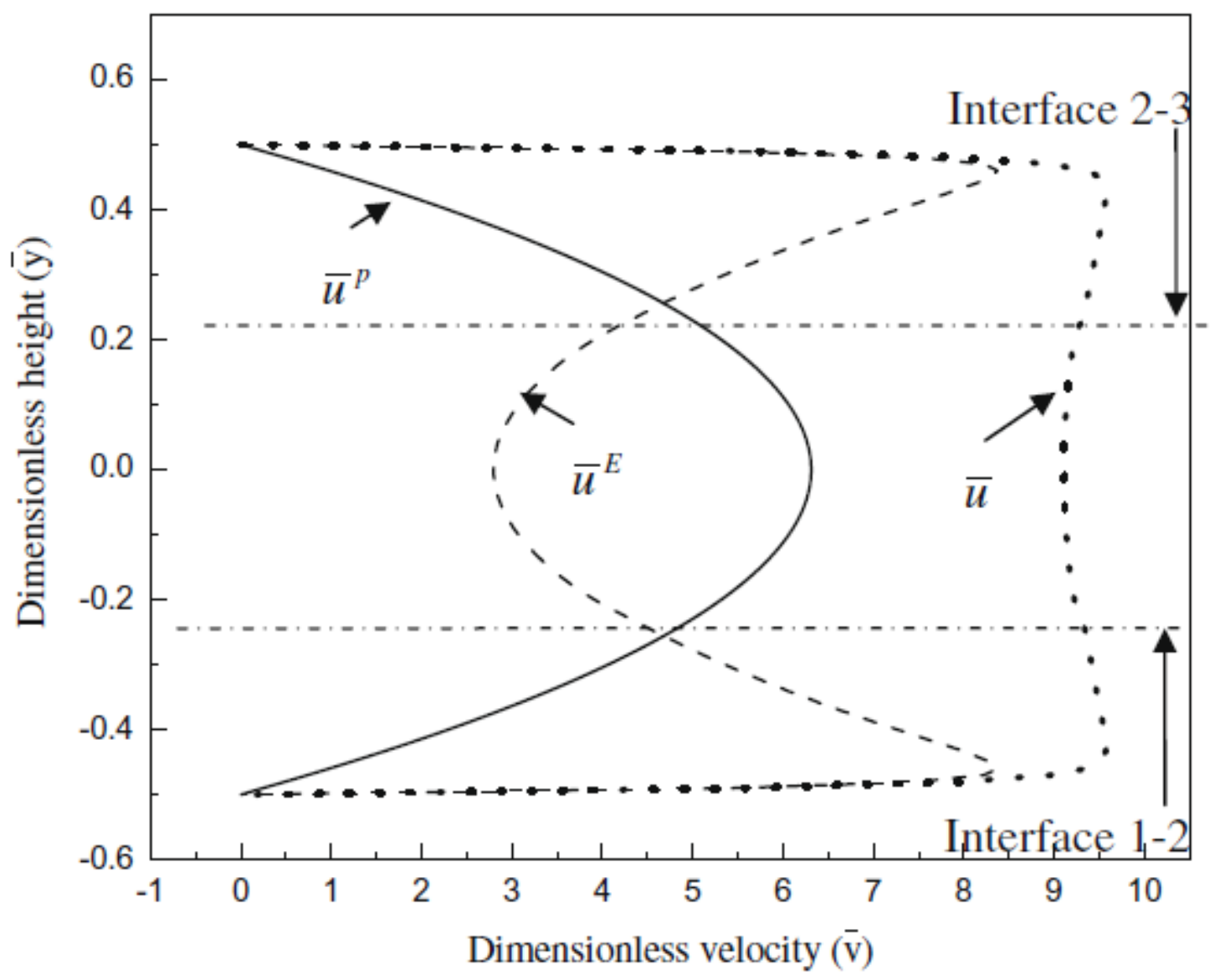

Fig. 5 


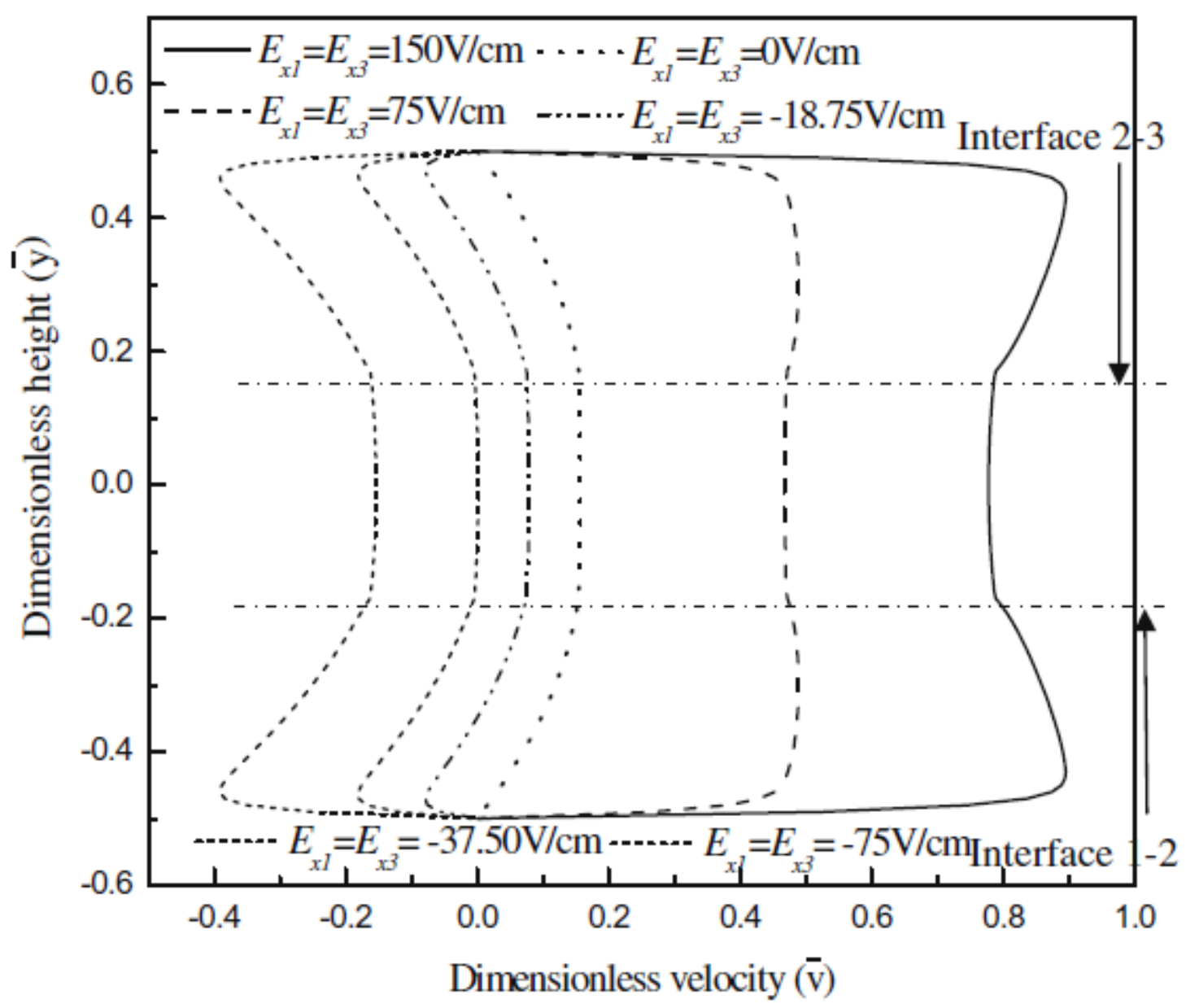

Fig. 6 


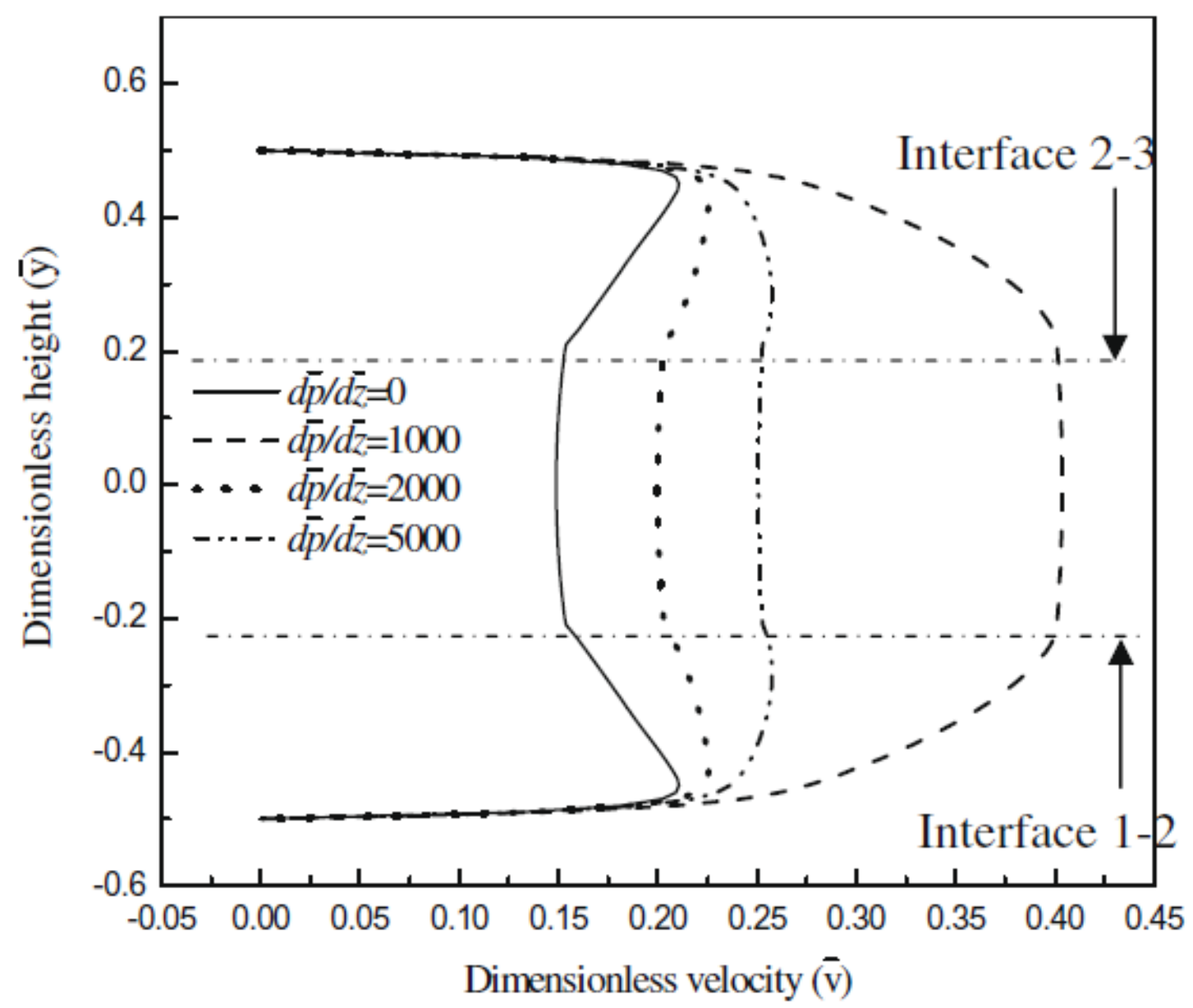

Fig. 7 


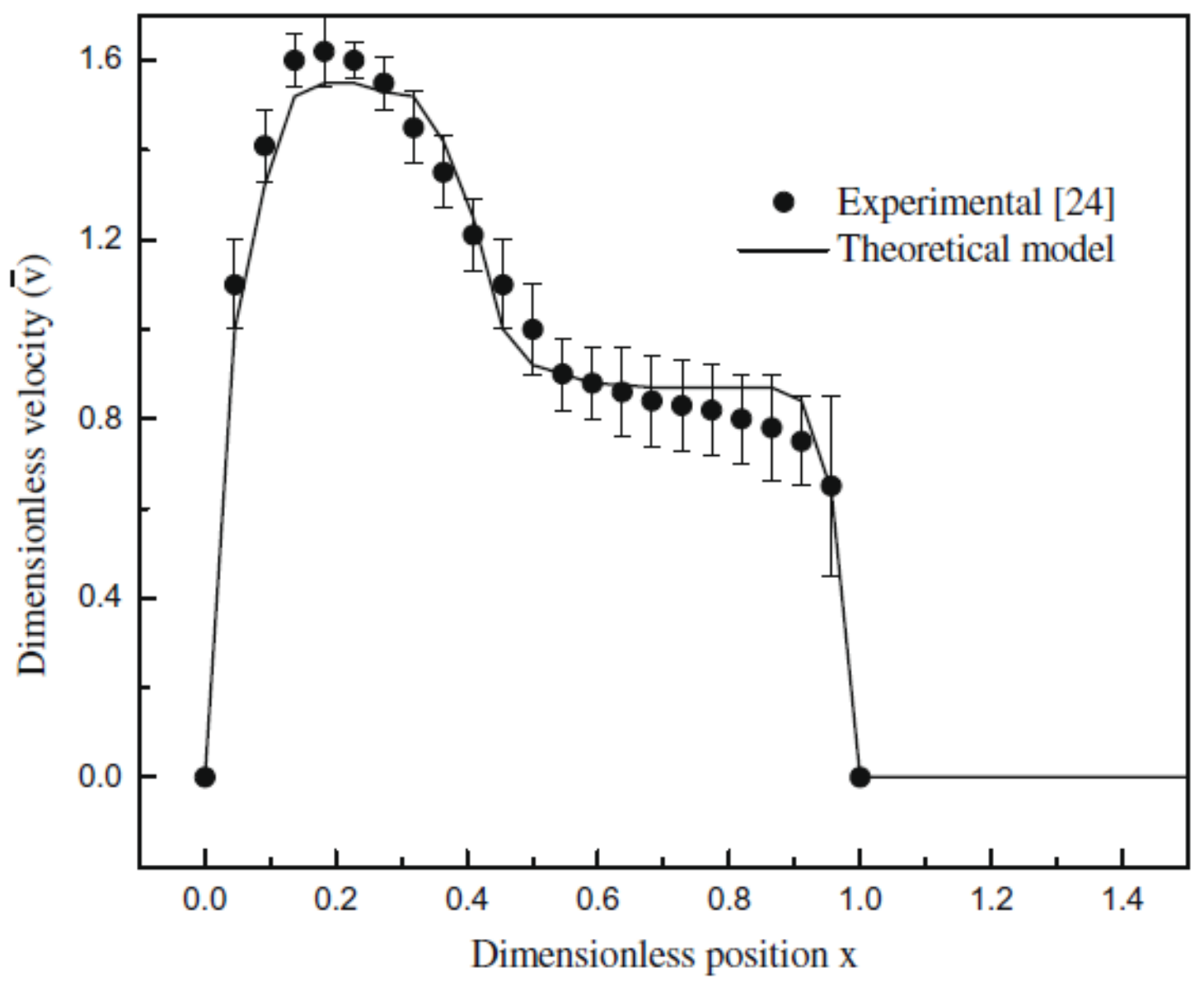

Fig. 8 


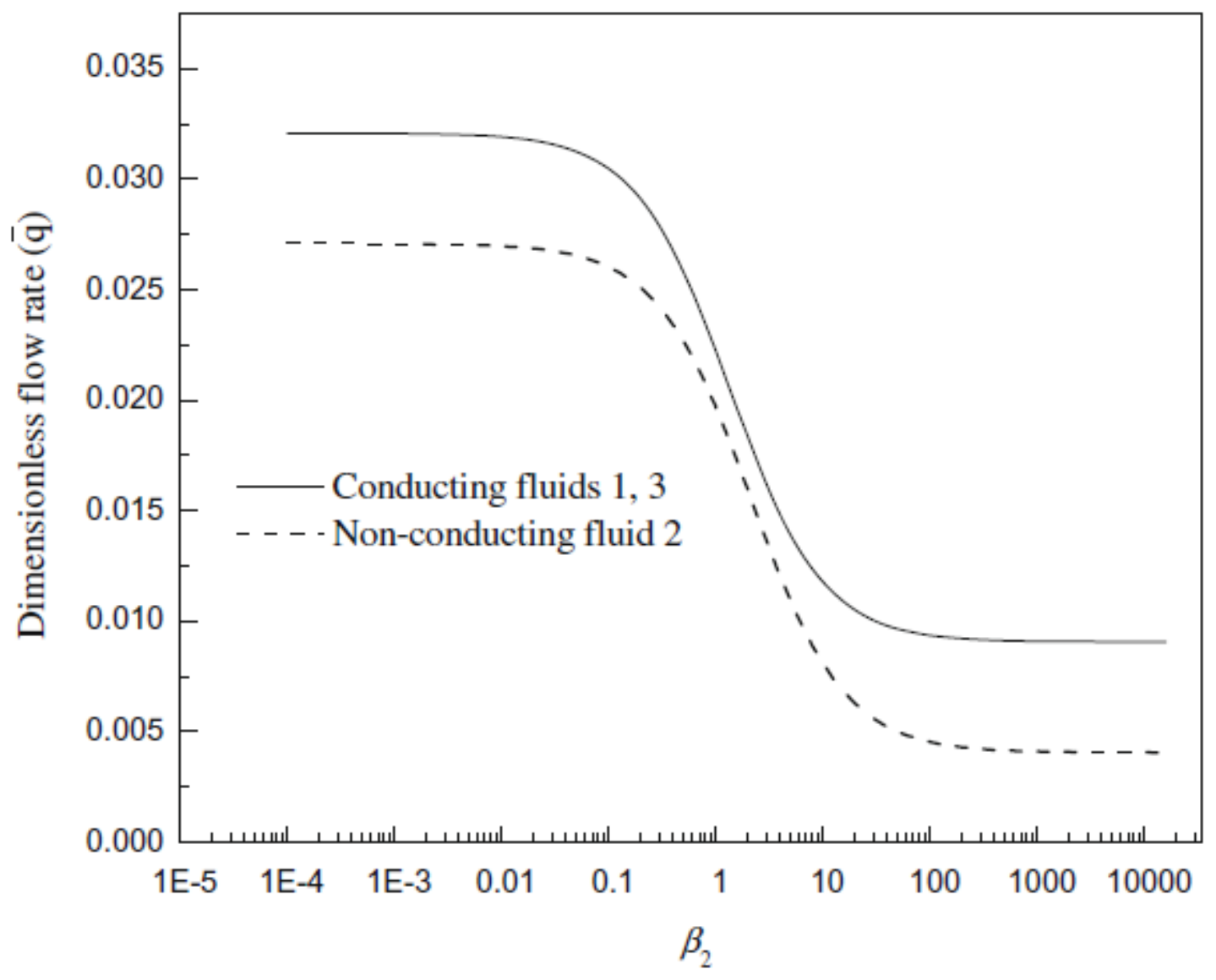

Fig. 9 